\title{
Global Existence and Asymptotic Stability to the Full 1D Hydrodynamic Model for Semiconductor Devices
}

\section{BO ZHANG}

\begin{abstract}
The full one-dimensional hydrodynamic model with small viscosity for semiconductor devices is studied. The model is selfconsistent in the sense that the electric field, which forms a forcing term in the momentum and energy equations, is determined by the coupled Poisson equation. Global existence is demonstrated for the model, which is shown to be equivalent to a non-standard integrodifferential equations. Finally, the asymptotic behavior of the smooth solution to the model is investigated.
\end{abstract}

1. Formulation and summary of results. We consider the following system of partial differential equations:

$$
\begin{aligned}
& \rho_{t}+v \rho_{x}=-v_{x} \rho, \\
& v_{t}+v v_{x}+\frac{1}{m \rho}(\rho T)_{x}-\frac{\varepsilon}{\rho} v_{x x}=\frac{e}{m} \varphi_{x}-\frac{v}{\tau_{p}}, \\
& T_{t}+v T_{x}+\frac{2}{3} T v_{x}-\frac{2}{3 \rho}\left(\kappa T_{x}\right)_{x}=\frac{m v^{2}\left(2 \tau_{w}-\tau_{p}\right)}{3 \tau_{p} \tau_{w}}-\frac{T-T^{(0)}}{\tau_{w}}, \\
& \frac{1}{e} \varphi_{x x}=\rho-C(x, t),
\end{aligned}
$$

where $\varepsilon$ is a positive parameter which measures the "viscosity."

We view the system of the equations (1)-(4) as a singular perturbation [5] of the full one-dimensional hydrodynamic equations for semiconductor devices in 
the case of one carrier type (e.g., electrons) [2, 9]:

$$
\left\{\begin{array}{l}
\rho_{t}+(\rho v)_{x}=0 \\
p_{t}+(p v+\rho T)_{x}=e \rho \varphi_{x}-\frac{p}{\tau_{p}} \\
W_{t}+(v W+\rho v T)_{x}-\left(\kappa T_{x}\right)_{x}=e \rho v \varphi_{x}-\frac{3 \rho\left(T-T^{(0)}\right)+m \rho v^{2}}{2 \tau_{w}} \\
\varphi_{x x}=e(\rho-C(x, t))
\end{array}\right.
$$

where $\rho(x, t), v(x, t), T(x, t)$, and $\varphi(x, t)$ denote the electron density, velocity, temperature, and electrostatic potential, respectively; $p$ and $W$ are called the momentum density and energy density, respectively. They are given by the following equations:

$$
\begin{aligned}
& p=m \rho v, \\
& W=\frac{3}{2} \rho T+\frac{1}{2} m \rho v^{2},
\end{aligned}
$$

where the positive constants $e$ and $m$ are the electronic charge and the effective electron mass, respectively. The dielectric constant has been set to 1 . The thermal conductivity $\kappa$ is given by a smooth function $\kappa=\kappa(\rho)>0$ on $\mathcal{R}^{+}$. The positive constant $T^{(0)}$ is the ambient device temperature; $\tau_{p}=\tau_{p}(\rho, v, T)$ and $\tau_{w}=\tau_{w}(\rho, v, T)$ are given functions on $\mathcal{R}^{+} \times \mathcal{R} \times \mathcal{R}^{+}$called the momentum and energy relaxation times. The device domain is the $x$-interval $(0,1)$. The given function $C(x, t)$ is the doping profile (background ion density).

The hydrodynamic model [2] for semiconductor devices plays an important role in simulating the behavior of the charge carrier in submicron semiconductor devices, since this model exhibits velocity overshoot which is not accounted for in the classical drift-diffusion model [1]. Some of its computational and physical aspects have been discussed by $[6,19]$. For steady-state subsonic electron flow in the full one-dimensional hydrodynamic model, Gardner et al. [11] proved the existence of solutions and the convergence of Newton's method. Also, Gardner [9] first simulated an electron shock wave in the case of steady-state of the full one-dimensional hydrodynamic model for submicrometer semiconductor devices. A mathematical analysis of a simplified one-dimensional hydrodynamic model for semiconductors, where the energy equation (3) is replaced by a pressure-density relationship, has been presented by Gamba [8] in steady-state and by Zhang [21] in evolution. In this paper we investigate the regularized system of equations (1)-(4), which is a set of compressible Navier-Stokes equations [3, 12, 15, 18] coupled to the Poisson equation. Novel elements in (2)-(3) include an electric force term and relaxation approximations to represent averaged collisions. Notice that the electric force term is nonlocal.

From a mathematical point of view, we prescribe the following impermeably insulated initial-boundary value conditions: 


$$
\begin{aligned}
& \left.(\rho, v, T)\right|_{t=0}=\left(\rho_{0}(x), v_{0}(x), T_{0}(x)\right), \quad x \in[0,1], \\
& v(0, t)=v(1, t)=0, \quad T_{x}(0, t)=T_{x}(1, t)=0, \quad t \geq 0, \\
& \varphi_{x}(0, t)=0, \quad \varphi(1, t)=\varphi_{1}, \quad t \geq 0,
\end{aligned}
$$

where $\varphi_{1}$ is the applied bias.

We give the following notations that are used in this paper. For an arbitrary time $S \in(0, \infty)$, set $Q=(0,1) \times(0, S]$ and let

$$
C^{n+\alpha, 1+\alpha / 2}(\bar{Q}):=\left\{f(x, t):\|f\|_{k+\alpha, \bar{Q}}=\sum_{i+n j=0}^{n}\left|\partial_{x}^{i} \partial_{t}^{j} f\right|_{\alpha, \bar{Q}}<\infty\right\}
$$

for $n=1,2,3$, where

$$
|f|_{\alpha, \bar{Q}}=\sup _{\bar{Q}}|f|+\sup _{(x, t),\left(x^{\prime}, t^{\prime}\right) \in \bar{Q}} \frac{\left|f(x, t)-f\left(x^{\prime}, t^{\prime}\right)\right|}{\left|x-x^{\prime}\right|^{\alpha}+\left|t-t^{\prime}\right|^{\alpha / 2}}, \quad 0<\alpha<1 .
$$

We also set

$$
H_{\alpha}(\bar{Q}):=\left\{f(x, t): f, f_{x} \in C^{\alpha, \alpha / 2}(\bar{Q})\right\}
$$

normed by

$$
\|f\|_{H_{\alpha}(\bar{Q})}=|f|_{\alpha, \bar{Q}}+\left|f_{x}\right|_{\alpha, \bar{Q}} .
$$

In order to obtain a solution of (1)-(4) with the desired regularity, we need the following assumptions:

(A $\left.\mathbf{A}_{1}\right)$ Relaxation times $\tau_{p}$ and $\tau_{w}$ satisfy uniform Lipschitz conditions on $\mathcal{R}^{+} \times \mathcal{R} \times \mathcal{R}^{+}, 0<\delta \leq \tau_{p}, \tau_{w} \leq L$ for some positive constants $\delta$ and $L$, and $0 \leq 2 \tau_{w}-\tau_{p} ; \kappa \in C^{2}\left(\mathcal{R}^{+}\right)$and $\delta \leq \kappa(\rho) \leq L$ on $\mathcal{R}^{+}$. Moreover, $C(x, t) \in C^{0}(\bar{Q})$.

$\left(\mathbf{A}_{2}\right) \rho_{0}(x) \in C^{1+\alpha}[0,1], v_{0}(x) \in C^{2+\alpha}[0,1], T_{0}(x) \in C^{2+\alpha}[0,1]$, $0<\delta \leq \rho_{0}(x) \leq L, \delta \leq T_{0}(x) \leq L$ on $[0,1]$. The initial-boundary value conditions satisfy the following compatibility conditions:

$$
v_{0}(i)=T_{0}^{\prime}(i)=0 \quad \text { for } i=0,1,
$$

where $(\cdot)^{\prime}=\frac{d}{d x}(\cdot)$. 
The assumptions on $\tau_{p}$ and $\tau_{w}$ are reasonable in semiconductor devices. For example, $\tau_{p}$ and $\tau_{w}$ satisfy the assumption $\left(\mathrm{A}_{1}\right)$ for a MOSFET (Metal Oxide Semiconductor Field Effect Transistor [10]) device.

The main results of this paper are as follows.

Theorem 1. Assume that $\left(\mathrm{A}_{1}\right)$ and $\left(\mathrm{A}_{2}\right)$ hold. Then, for an arbitrary time $S \in(0, \infty)$, there exists a unique solution $(\rho, v, T, \varphi)$ of the initial-boundary value problem (1)-(7) satisfying

$$
\begin{gathered}
\rho \in C^{1+\alpha, 1+\alpha / 2}(\bar{Q}), \quad v \in C^{2+\alpha, 1+\alpha / 2}(\bar{Q}), \\
T \in C^{2+\alpha, 1+\alpha / 2}(\bar{Q}), \quad \varphi \in C^{3+\alpha, 1+\alpha / 2}(\bar{Q}), \\
\rho(x, t)>0, \quad T(x, t)>0, \quad(x, t) \in \bar{Q} .
\end{gathered}
$$

Theorem 2. Assume that the conditions of Theorem 1 hold and that $\rho_{0}(x)=$ $C(x)$ for $x \in[0,1]$. Then, the solution $(\rho, v, T, \varphi)$ of the initial-boundary problem (1)-(7) is asymptotically stable in the following sense:

$$
\begin{array}{ll}
\|\rho\|_{L^{1}(0,1)}(t)=\left\|\rho_{0}\right\|_{L^{1}(0,1)}, & t \geq 0, \\
v^{2}(x, t) \rightarrow 0, x \in[0,1], & t \rightarrow \infty, \\
T(x, t) \rightarrow T^{(0)}, x \in[0,1], & t \rightarrow \infty, \\
\left\|\varphi_{x}\right\|_{L^{2}(0,1)}^{2}(t)=f(t), & t \geq 0,
\end{array}
$$

where $f(t)=\int_{0}^{1}\left(\int_{0}^{t} \rho v d t\right)^{2} d x$ satisfying $f(\infty)<\infty$.

The plan of this paper is as follows. In Section 2 we reduce the system of the equations (1)-(4) to a pair of integro-differential equations (29)-(30) by using the characteristics method. We obtain a priori estimates by applying the energy method and techniques used in Section 3. We then show the existence of a globally smooth solution of (29)-(30) by using the Leray-Schauder fixed point theorem in Section 4, thereby proving Theorem 1. In Section 5 we show that the solution of (1)-(4) converges to a steady-state solution as $t \rightarrow \infty$.

2. Characteristic method. In this section we use the characteristic method $[13,20]$ to reduce the system of equations (1)-(4) to a system of parabolic equations. Consider the following characteristic equation for (1) as a linear equation with respect to $\rho$ :

$$
\left\{\begin{array}{l}
\frac{d}{d \sigma} \bar{y}(\sigma ; x, t)=v(\bar{y}(\sigma ; x, t), \sigma), \quad 0 \leq \sigma \leq t \leq S, \\
\bar{y}(t ; x, t)=x .
\end{array}\right.
$$


Let $\bar{y}(\sigma ; x, t)$ be the solution of (12). We then have a coordinate transformation as follows:

$$
y=y(x, t) \equiv \bar{y}(0 ; x, t), \quad s=s(x, t) \equiv t .
$$

This transformation $(x, t) \mapsto(y(x, t), s)$ is a one-to-one mapping from $\bar{Q}$ onto itself and keeps the same boundaries $\{0\} \times[0, T] \cup\{1\} \times[0, T]$ since $v(0, t)=$ $v(1, t)=0$. We use $(y, s)$ as the characteristic coordinate, $x$ and $t$ are the inverse expressed by

$$
x=x(y, s) \equiv \bar{y}(s ; y, 0), \quad s=s(x, t) \equiv t .
$$

For the function $v(x, t)$, we introduce a notation $\hat{v}(y, s) \equiv v(x(y, s), s)$, then

$$
\left\{\begin{array}{l}
\frac{d}{d s} x(y, s)=\hat{v}(y, s) \\
x(y, 0)=y
\end{array} .\right.
$$

It follows that

$$
x(y, s)=y+\int_{0}^{s} \hat{v}(y, \sigma) d \sigma .
$$

Then,

$$
\frac{\partial x}{\partial y}=1+\int_{0}^{s} \hat{v}_{y}(y, \sigma) d \sigma .
$$

For simplicity, we denote

$$
\theta=\theta(\hat{v}) \equiv \int_{0}^{s} \hat{v}_{y}(y, \sigma) d \sigma .
$$

Differentiating (12) with respect to $x$, we have

$$
\left\{\begin{array}{l}
\frac{d}{d \sigma} \bar{y}_{x}(\sigma ; x, t)=v_{x}(\bar{y}(\sigma ; x, t)) \bar{y}_{x}(\sigma ; x, t), \\
\bar{y}_{x}(t ; x, t)=1 .
\end{array}\right.
$$

Then,

$$
\bar{y}_{x}(0 ; x, t)=e^{-\int_{0}^{t} v_{x}(\bar{y}(\sigma ; x, t) d \sigma} .
$$

By (13) and (16), we have

$$
\frac{\partial y}{\partial x}=\frac{1}{1+\theta}=e^{-\int_{0}^{t} v_{x}(\bar{y}(\sigma ; x, t) d \sigma}>0 .
$$


Let $(x, t)=(x(y, s), s)$. From the chain rule, the system of equations (1)-(4) reads:

$$
\begin{aligned}
& \hat{\rho}=-\frac{\hat{v}_{y}}{1+\theta} \hat{\rho}, \\
& \hat{v}_{s}+\frac{1}{m \hat{\rho}(1+\theta)}(\hat{\rho} \hat{T})_{y}-\frac{\varepsilon}{\hat{\rho}(1+\theta)}\left(\frac{\hat{v}_{y}}{1+\theta}\right)_{y}=\frac{e}{m} \frac{\hat{\varphi}_{y}}{1+\theta}-\frac{\hat{v}}{\tau_{p}}, \\
& \hat{T}_{s}+\frac{2}{3(1+\theta)} \hat{v}_{y} \hat{T}-\frac{2}{3 \hat{\rho}(1+\theta)}\left(\kappa \frac{\hat{T}_{y}}{1+\theta}\right)_{y} \\
& \quad=\frac{m \hat{v}^{2}\left(2 \tau_{w}-\tau_{p}\right)}{3 \tau_{p} \tau_{w}}-\frac{\hat{T}-T^{(0)}}{\tau_{w}}, \\
& \frac{1}{1+\theta}\left(\frac{\varphi_{y}}{1+\theta}\right)_{y}=e(\rho-C) .
\end{aligned}
$$

The initial-boundary value conditions (5)-(7) are translated into similar conditions:

$$
\begin{aligned}
& \left.(\hat{\rho}, \hat{v}, \hat{T})\right|_{s=0}=\left(\rho_{0}(y), v_{0}(y), T_{0}(y)\right), \quad y \in[0,1], \\
& \hat{v}(0, s)=\hat{v}(1, s)=0, \quad \hat{T}_{y}(0, s)=\hat{T}_{y}(1, s)=0, \quad s \geq 0, \\
& \hat{\varphi}_{y}(0, s)=0, \quad \hat{\varphi}(1, s)=\varphi_{1}(s), \quad s \geq 0 .
\end{aligned}
$$

There exists a unique solution of (18) and (22):

$$
\hat{\rho}(y, s)=\frac{1}{1+\theta} \rho_{0}(y) .
$$

Substituting (25) for $\hat{\rho}$ in (21) and solving (21) and (24), we have

$$
\frac{\hat{\varphi}_{y}}{1+\theta}=e\left(\bar{\rho}_{0}-D\right)
$$

where $\bar{\rho}_{=} \int_{0}^{y} \rho_{0}(\xi) d \xi$ and $D=\int_{0}^{y} C(\xi, s) d \xi$.

Finally, substituting (25) and (26) for $\hat{\rho}$ and $\hat{\varphi}_{y} /(1+\theta)$ in the equations (19)-(20), we obtain

$$
\begin{aligned}
& \hat{v}_{s}+\frac{1}{m \rho_{0}}\left(\frac{f_{0}}{1+\theta} \hat{T}\right)_{y}-\frac{\varepsilon}{\rho_{0}}\left(\frac{\hat{v}_{y}}{1+\theta}\right)_{y}=\frac{e^{2}}{m}\left(\bar{\rho}_{0}-D\right)-\frac{\hat{v}}{\tau_{p}} \\
& \hat{T}_{s}+\frac{2 \hat{v}_{y} \hat{T}}{3(1+\theta)}-\frac{2}{3 \rho_{0}}\left(\frac{\kappa \hat{T}_{y}}{1+\theta}\right)_{y}=\frac{m \hat{v}^{2}\left(2 \tau_{w}-\tau_{p}\right)}{3 \tau_{p} \tau_{w}}-\frac{\hat{T}-T^{(0)}}{\tau_{w}} .
\end{aligned}
$$


Thus, the system of equations (1)-(4) is reduced to a system of nonlinear integro-differential equations $(27)-(28)$. Since $1 /(1+\theta)>0$, we may visualize the integro-differential equations (27)-(28) as a couple of parabolic equations. For simplicity, we drop the hat "»" in the subsequent sections.

3. A priori estimates. We consider the following initial-boundary value problems in the region $Q=(0,1) \times(0, S]$ :

$$
\begin{aligned}
& v_{s}+\frac{1}{m \rho_{0}}\left(\frac{\rho_{0}}{1+\theta} T\right)_{y}-\frac{\varepsilon}{\rho_{0}}\left(\frac{v_{y}}{1+\theta}\right)_{y}=\frac{e^{2}}{m}\left(\bar{\rho}_{0}-D\right)-\frac{v}{\tau_{p}}, \\
& T_{s}+\frac{2 v_{y} T}{3(1+\theta)}-\frac{2}{3 \rho_{0}}\left(\frac{\kappa T_{y}}{1+\theta}\right)_{y}=\frac{m v^{2}\left(2 \tau_{w}-\tau_{p}\right)}{3 \tau_{p} \tau_{w}}-\frac{T-T^{(0)}}{\tau_{w}}, \\
& v(y, 0)=v_{0}(y), \quad T(y, 0)=T_{0}(y), \\
& v(0, s)=v(1, s)=0, \quad T_{y}(0, s)=T_{y}(1, s)=0,
\end{aligned}
$$

Let $\{v, T\}$ be a solution of (29)-(32). From the energy method, we have the following estimates.

Lemma 1. There is a positive constant $M_{1}$ depending only on initial data such that

$$
\begin{aligned}
& \int_{0}^{1} \rho_{0} v^{2} d y+\frac{3}{m} \int_{0}^{1} \rho_{0} T d y \leq M_{1}, \\
& \varepsilon \int_{0}^{s} \int_{0}^{1} \frac{v_{y}^{2}}{1+\theta} d y d s \leq M_{1} .
\end{aligned}
$$

Proof. Multiplying (29) and (30) by $\rho_{0} v$ and $\frac{3}{2 m} \rho_{0}$, respectively, and integrating over $(0,1)$, we have

$$
\begin{aligned}
& \frac{1}{2} \frac{d}{d s} \int_{0}^{1} \rho_{0} v^{2} d y-\frac{1}{m} \int_{0}^{1} \frac{\rho_{0} v_{y} T}{1+\theta} d y+\int_{0}^{1}\left(\frac{\varepsilon v_{y}^{2}}{1+\theta}+\frac{\rho_{0} v^{2}}{\tau_{p}}\right) d y \\
= & \frac{e^{2}}{m} \int_{0}^{1} \rho_{0}\left(\bar{\rho}_{0}-D\right) v d y \\
& \frac{3}{2 m} \frac{d}{d s} \int_{0}^{1} \rho_{0} T d y+\frac{1}{m} \int_{0}^{1} \frac{\rho_{0} v_{y} T}{1+\theta} d y-\int_{0}^{1} \frac{\rho_{0} v^{2}\left(2 \tau_{w}-\tau_{p}\right)}{2 \tau_{p} \tau_{w}} d y \\
= & -\frac{3}{2 m} \int_{0}^{1} \frac{\rho_{0}\left(T-T^{(0)}\right)}{\tau_{w}} d y .
\end{aligned}
$$


Adding (36) to (35), we obtain

$$
\begin{gathered}
\frac{1}{2} \int_{0}^{1} \rho_{0}\left(v^{2}+\frac{3}{m} T\right) d y+\int_{0}^{s} \int_{0}^{1}\left(\frac{\varepsilon v_{y}^{2}}{1+\theta}+\frac{\rho_{0} v^{2}}{2 \tau_{w}}+\frac{3 \rho_{0} T}{2 m \tau_{w}}\right) d y d s \\
=\frac{1}{2} \int_{0}^{1} \rho_{0}\left(v_{0}^{2}+\frac{3}{m} T_{0}\right) d y+\frac{3 T^{(0)}}{2 m} \int_{0}^{s} \int_{0}^{1} \frac{d y d s}{\tau_{w}} \\
+\frac{e^{2}}{m} \int_{0}^{s} \int_{0}^{1} \rho_{0}\left(\bar{\rho}_{0}-D\right) v d y d s .
\end{gathered}
$$

The following, slightly weaker, inequality follows from (37):

$$
\begin{aligned}
& \frac{1}{2} \int_{0}^{1} \rho_{0}\left(v^{2}+\frac{3}{m} T\right) d y+\int_{0}^{s} \int_{0}^{1} \frac{\varepsilon v_{y}^{2}}{1+\theta} d y d s \\
&+\frac{1}{4} \int_{0}^{s} \int_{0}^{1} \rho_{0}\left(\frac{\rho_{0} v^{2}}{\tau_{w}}+\frac{6 \rho_{0} T}{m \tau_{w}}\right) d y d s \\
& \leq \frac{1}{2} \int_{0}^{1} \rho_{0}\left(v_{0}^{2}+\frac{3}{m} T_{0}\right) d y+\frac{3 T^{(0)}}{2 m} \int_{0}^{s} \int_{0}^{1} \frac{d y d s}{\tau_{w}} \\
&+\frac{e^{2}}{m} \int_{0}^{s} \int_{0}^{1} \rho_{0}\left(\bar{\rho}_{0}-D\right)^{2} \tau_{w} d y d s \equiv M_{1} .
\end{aligned}
$$

Therefore, (33) and (34) hold.

Lemma 2. There exists a positive constant $M_{2}$ independent of $\varepsilon$ such that

$$
\begin{gathered}
\int_{0}^{1} \rho_{0}[(1+\theta)-1-\ln (1+\theta)] d y+\int_{0}^{1} \rho_{0}(T-1-\ln T) d y \\
+\int_{0}^{s} \int_{0}^{1} \frac{\kappa T_{y}^{2}}{(1+\theta) T^{2}} d y d s \leq M_{2}
\end{gathered}
$$

Proof. First, note that

$$
\begin{aligned}
\frac{1}{m} \int_{0}^{1} \rho_{0} \theta d y & =\frac{1}{m} \int_{0}^{1} \rho_{0} \int_{0}^{s} v_{y} d \sigma d y \\
& \leq \frac{2}{m^{2}} \int_{0}^{s} \int_{0}^{1} \frac{\left(\rho_{0}^{\prime}\right)^{2} \tau_{w}}{\rho_{0}} d y d s+\frac{1}{8} \int_{0}^{s} \int_{0}^{1} \frac{\rho_{0} v^{2}}{\tau_{w}} d y d s
\end{aligned}
$$


Now, multiplying $(30)$ by $3 \rho_{0} /(2 m T)$ and integrating over $(0,1) \times(0, s)$, we have

$$
\begin{aligned}
& \frac{1}{m} \int_{0}^{1} \rho_{0}\left(\ln (1+\theta)+\frac{3}{2} \ln T\right) d y-\frac{1}{m} \int_{0}^{s} \int_{0}^{1} \frac{\kappa T_{y}^{2}}{(1+\theta) T^{2}} d y d s \\
= & \int_{0}^{s} \int_{0}^{1}\left(\frac{\rho_{0} v^{2}\left(2 \tau_{w}-\tau_{p}\right)}{2 \tau_{p} \tau_{w} T}-\frac{3 \rho_{0}\left(T-T^{(0)}\right)}{2 m \tau_{w} T}\right) d y d s+\frac{3}{2 m} \int_{0}^{1} \rho_{0} \ln T_{0} d y .
\end{aligned}
$$

Subtracting (40) from (38), we obtain

$$
\begin{aligned}
& \frac{3}{2 m} \int_{0}^{1} \rho_{0}(T-1-\ln T) d y+\frac{1}{m} \int_{0}^{1} \rho_{0}[(1+\theta)-1-\ln (1+\theta)] d y \\
& +\frac{1}{2} \int_{0}^{1} \rho_{0} v^{2} d y+\int_{0}^{s} \int_{0}^{1}\left(\frac{\varepsilon v_{y}^{2}}{1+\theta}+\frac{\kappa T_{y}^{2}}{m(1+\theta) T^{2}}\right) d y d s \\
& +\int_{0}^{s} \int_{0}^{1}\left(\frac{\rho_{0} v^{2}}{4 \tau_{w}}+\frac{3 \rho_{0} T}{2 m \tau_{w}}+\frac{\rho_{0} v^{2}\left(2 \tau_{w}-\tau_{p}\right)}{2 \tau_{p} \tau_{w} T}+\frac{3 \rho_{0} T^{(0)}}{2 m \tau_{w} T}\right) d y d s \\
& \leq \quad M_{1}+\frac{3}{2 m} \int_{0}^{s} \int_{0}^{1} \frac{\rho_{0}}{\tau_{w}} d y d s+\frac{2}{m^{2}} \int_{0}^{s} \int_{0}^{1} \frac{\left(\rho_{0}^{\prime}\right)^{2} \tau_{w}}{\rho_{0}} d y d s \\
& \quad-\frac{3}{2 m} \int_{0}^{1} \rho_{0} \ln T_{0} d y+\frac{1}{8} \int_{0}^{s} \int_{0}^{1} \frac{\rho_{0} v^{2}}{\tau_{w}} d y d s \\
& \equiv M_{2}+\frac{1}{8} \int_{0}^{s} \frac{\rho_{0} v^{2}}{\tau_{w}} d y d s .
\end{aligned}
$$

Notice that the function $h(z)=z-1-\ln z$ is nonnegative for $z \in \mathcal{R}^{+}$. It follows from (41) that (39) holds.

Lemma 3. There exists a positive constant $M_{3}$ depending on $\varepsilon$ such that

$$
\frac{1}{M_{3}} \leq 1+\theta \leq M_{3}
$$

Proof. We consider the function $F(y, s)$ defined by

$$
F(y, s)=\frac{1}{\varepsilon} \int_{0}^{y} \rho_{0}(\xi)\left(v_{0}(\xi)-v(\xi, s)\right) d \xi+\left.\int_{0}^{s}\left(\frac{\rho_{0} T}{\varepsilon m(1+\theta)}-\frac{v_{y}}{1+\theta}\right)\right|_{y=0} d \sigma
$$


Then,

$$
F_{s}=-\frac{v_{y}}{1+\theta}+\frac{1}{\varepsilon m} \frac{\rho_{0} T}{1+\theta}+g,
$$

where

$$
g=\frac{1}{\varepsilon} \int_{0}^{y} \rho_{0}\left(\frac{v}{\tau_{p}}-\frac{e^{2}}{m}\left(\bar{\rho}_{0}-D\right)\right) d y .
$$

From the fact that

$$
v_{y}=(1+\theta)_{s}
$$

and (43), we have the following ordinary differential equation:

$$
\left\{\begin{array}{l}
\frac{d}{d s}(1+\theta)+\left(F_{s}-g\right)(1+\theta)=\frac{1}{\varepsilon m} \rho_{0} T, \\
\left.(1+\theta)\right|_{s=0}=1 .
\end{array}\right.
$$

It follows that

$$
1+\theta=e^{-F(y, s)+\int_{0}^{s} g d s}\left(1+\frac{\rho_{0}}{\varepsilon m} \int_{0}^{s} T e^{F(y, s)-\int_{0}^{s} g d \sigma} d s\right) .
$$

In order to get (42), we first estimate $F(y, s)$ and $\int_{0}^{s} g d \sigma$. By Lemma 1, we have

$$
\begin{gathered}
\sup \left|\int_{0}^{s} g d \sigma\right| \leq C(\varepsilon), \\
|F(y, s)-F(1, s)|=\frac{1}{\varepsilon}\left|\int_{y}^{1} \rho_{0}\left(v_{0}-v\right) d y\right| \leq C(\varepsilon) .
\end{gathered}
$$

We claim that $|F(1, s)|$ is bounded. In fact, it follows from (44) and Lemma 1 that

$$
\begin{aligned}
\int_{0}^{s} \int_{0}^{1} & {\left[\frac{d}{d s}(1+\theta)+(1+\theta) F_{s}\right] d y d s } \\
& =\int_{0}^{s} \int_{0}^{1}\left[\frac{1}{\varepsilon m} \rho_{0} T+g(1+\theta)\right] d y d s \\
& =\int_{0}^{s} \int_{0}^{1}\left(\frac{1}{\varepsilon m} \rho_{0} T+g-g_{y} \int_{0}^{s} v d \sigma\right) d y d s \leq C(\varepsilon) .
\end{aligned}
$$

The left-hand side of (46) can be rewritten as follows: 


$$
\begin{aligned}
& \int_{0}^{s} \int_{0}^{1} {\left[\frac{d}{d s}(1+\theta)+(1+\theta) F_{s}\right] d y d s } \\
&=\int_{0}^{s} \int_{0}^{1} v_{y}(1-F) d y d s+\int_{0}^{1}(1+\theta) F d y \\
&=\int_{0}^{1} F d y+\int_{0}^{1}\left(\int_{0}^{s} v d s\right)_{y} F d y+\int_{0}^{s} \int_{0}^{1} v F_{y} d y d s \\
&=F(1, s)-\frac{1}{\varepsilon} \int_{0}^{1} \rho_{0}\left(v_{0}-v\right)\left(y+\int_{0}^{s} v d s\right) d y \\
& \quad+\frac{1}{\varepsilon} \int_{0}^{s} \int_{0}^{1} \rho_{0} v\left(v_{0}-v\right) d y d s .
\end{aligned}
$$

It follows from (46)-(47) and Lemma 1 that

$$
|F(1, s)| \leq C(\varepsilon), \quad 0 \leq s \leq S .
$$

Thus,

$$
\sup _{\bar{Q}}|F(y, s)| \leq \sup _{\bar{Q}}|F(y, s)-F(1, s)|+\sup _{0 \leq s \leq 1}|F(1, s)| \leq C(\varepsilon) .
$$

It follows from (45) and (48) that

$$
1+\theta \geq e^{-C(\varepsilon)}\left(1+\frac{\rho_{0}}{\varepsilon m} \int_{0}^{s} e^{-C(\varepsilon)} T d s\right) \geq e^{-C(\varepsilon)} .
$$

Similarly, we obtain the upper bound of $1+\theta$. By Lemma 2, for every $s \in[0, S]$, there exists a $y(s) \in[0,1]$ such that

$$
\left.R_{1} \leq T(y(s), s)\right) \leq R_{2},
$$

where $R_{1}$ and $R_{2}$ are the two positive roots of the equation $z-1-\ln z=M_{2} / \delta$. Then,

$$
\text { (49) } \begin{aligned}
\sqrt{T(y, s)} & =\sqrt{T(y(s), s)}+\frac{1}{2} \int_{y(s)}^{y} \frac{T_{y}}{\sqrt{T}} d y \\
& \leq \sqrt{R_{2}}+\frac{1}{2} \int_{0}^{1}\left|\frac{\sqrt{\kappa} T_{y}}{\sqrt{1+\theta} T} \frac{\sqrt{1+\theta} \sqrt{T}}{\sqrt{\kappa}}\right| d y \leq
\end{aligned}
$$




$$
\begin{aligned}
& \leq \sqrt{R_{2}}+\frac{1}{2}\left(\int_{0}^{1} \frac{\kappa T_{y}^{2}}{(1+\theta) T^{2}} d y\right)^{1 / 2}\left(\int_{0}^{1} \frac{(1+\theta) T}{\kappa} d y\right)^{1 / 2} \\
& \leq \sqrt{R_{2}}+\frac{1}{2}\left(\frac{M_{1}}{\delta} U(s) \max _{0 \leq y \leq 1}(1+\theta)\right)^{1 / 2} \quad \text { (Lemma 1), }
\end{aligned}
$$

where

$$
U(s)=\int_{0}^{1} \frac{\kappa T_{y}^{2}}{(1+\theta) T^{2}} d y .
$$

It follows from (45) and (49) that there are two positive constants $N_{1}$ and $N_{2}$ depending on $\varepsilon$ such that

$$
\max _{0 \leq y \leq 1}(1+\theta) \leq N_{1}+N_{2} \int_{0}^{s} U(s) \max _{0 \leq y \leq 1}(1+\theta) d s .
$$

By Gronwall's inequality and Lemma 2, we have

$$
\max _{0 \leq y \leq 1}(1+\theta) \leq N_{1} e^{N_{2} \int_{0}^{S} U(s) d s} \leq N_{1} e^{C_{2} M_{2}} .
$$

Let $M_{3}=\max \left\{N_{1} e^{N_{2} M_{2}}, e^{C(\varepsilon)}\right\}$, then (42) holds.

Lemma 4. There exists a positive constant $M_{4}$ depending on $\varepsilon$ such that

$$
\int_{0}^{1}\left(v^{4}+T^{2}\right) d y+\int_{0}^{s} \int_{0}^{1}\left(v^{2} v_{y}^{2}+T_{y}^{2}\right) d y d s \leq M_{4} .
$$

Proof. From (29) and (30), we have

$$
\begin{aligned}
& \left(\frac{1}{2} \rho_{0} v^{2}+\frac{3}{2 m} \rho_{0} T\right)_{s}+\frac{1}{m}\left(\frac{\rho_{0}}{1+\theta} v T\right)_{y}-\varepsilon\left(\frac{v_{y}}{1+\theta}\right)_{y} v-\frac{1}{m}\left(\frac{\kappa}{1+\theta} T_{y}\right)_{y} \\
= & \frac{e^{2}}{m} \rho_{0} v\left(\bar{\rho}_{0}-D\right)-\frac{\rho_{0} v^{2}}{2 \tau_{w}}-\frac{3 \rho_{0} T}{2 m \tau_{w}}+\frac{3 \rho_{0} T^{(0)}}{2 m \tau_{w}} .
\end{aligned}
$$

Multiplying the equality above by

$$
\left(\frac{1}{2} \rho_{0} v^{2}+\frac{3}{2 m} \rho_{0} T\right)
$$


and using integration by parts, we obtain

$$
\begin{aligned}
& \frac{1}{2} \frac{d}{d s} \int_{0}^{1}\left(\frac{1}{2} \rho_{0} v^{2}+\frac{3}{2 m} \rho_{0} T\right)^{2} d y-\frac{1}{m} \int_{0}^{1} \frac{\rho_{0} v T}{1+\theta}\left(\frac{1}{2} \rho_{0} v^{2}+\frac{3}{2 m} \rho_{0} T\right)_{y} d y \\
+ & \varepsilon \int_{0}^{1} \frac{v_{y}}{1+\theta}\left(\frac{1}{2} \rho_{0} v^{3}+\frac{3}{2 m} \rho_{0} v T\right)_{y} d y+\frac{1}{m} \int_{0}^{1} \frac{\kappa T_{y}}{1+\theta}\left(\frac{1}{2} \rho_{0} v^{2}+\frac{3}{2 m} \rho_{0} T\right)_{y} d y \\
= & \int_{0}^{1}\left[\frac{e^{2}}{m} \rho_{0} v\left(\bar{\rho}_{0}-D\right)-\frac{\rho_{0} v^{2}}{2 \tau_{w}}-\frac{3 \rho_{0} T}{2 m \tau_{w}}+\frac{3 \rho_{0} T^{(0)}}{2 m \tau_{w}}\right]\left(\frac{1}{2} \rho_{0} v^{2}+\frac{3}{2 m} \rho_{0} T\right) d y .
\end{aligned}
$$

Differentiate out the terms in the integrals, use the bounds on $\rho_{0}$ and $1+\theta$, and apply Lemma 1, along with the usual inequalities for the product of two terms. After this tedious calculation, it can be seen that

$$
\begin{aligned}
& \int_{0}^{1}\left(v^{4}+T^{2}\right) d y+\int_{0}^{s} \int_{0}^{1}\left(v^{2} v_{y}^{2}+T_{y}^{2}+T v_{y}^{2}\right) d y d s \\
\leq & N_{1}+N_{2} \int_{0}^{s} \int_{0}^{1}\left[v^{2} v_{y}^{2}+v^{4}+(1+|v|)^{2} T^{2}\right] d y,
\end{aligned}
$$

where $N_{1}$ and $N_{2}$ depend on $\varepsilon$ and the data.

Next, multiply $(29)$ by $\rho_{0} v^{3}$ and integrate it over $(0,1)$ to see that

$$
\begin{aligned}
& \frac{1}{4} \int_{0}^{1} \rho_{0} v^{4} d y+\int_{0}^{s} \int_{0}^{1}\left(\frac{3 \varepsilon v^{2} v_{y}^{2}}{1+\theta}-\frac{3 \rho_{0} T v^{2} v_{y}}{m(1+\theta)}+\frac{\rho_{0} v^{4}}{\tau_{p}}\right) d y d s \\
= & \int_{0}^{1} \rho_{0} v_{0}^{4} d y+\frac{e^{2}}{m} \int_{0}^{s} \int_{0}^{1} \rho_{0} v^{3}\left(\bar{\rho}_{0}-D\right) d y d s .
\end{aligned}
$$

By a similar argument, there exist positive constants $N_{3}$ and $N_{4}$ depending on $\varepsilon$ and the data such that

$$
\int_{0}^{1} v^{4} d y+\int_{0}^{s} \int_{0}^{1} v^{2} v_{y}^{2} d y d s \leq N_{3}+N_{4} \int_{0}^{s} \int_{0}^{1}\left(v^{4}+v^{2} T^{2}\right) d y d s .
$$

It follows from (51) and (52) that

$$
\begin{aligned}
& \int_{0}^{1}\left(v^{4}+T^{2}\right) d y+\int_{0}^{s} \int_{0}^{1}\left(v^{2} v_{y}^{2}+T_{y}^{2}+T v_{y}^{2}\right) d y d s \\
\leq & N_{5}+N_{6} \int_{0}^{s} \int_{0}^{1}\left(v^{4}+\left(1+v^{2}\right) T^{2}\right) d y d s .
\end{aligned}
$$


Recall the interpolation inequality

$$
T^{2} \leq \beta \int_{0}^{1} T_{y}^{2} d y+C_{\beta} \int_{0}^{1} T^{2} d y .
$$

As a consequence of it, (53), and Lemma 1, we have

$$
\begin{aligned}
& \int_{0}^{1}\left(v^{4}+T^{2}\right) d y+\int_{0}^{s} \int_{0}^{1}\left(v^{2} v_{y}^{2}+T_{y}^{2}+T v_{y}^{2}\right) d y d s \\
\leq & N_{7}+N_{8} \int_{0}^{s} \int_{0}^{1}\left(v^{4}+T^{2}\right) d y d s .
\end{aligned}
$$

It follows from Gronwall's inequality that (50) holds.

Lemma 5. There exists a positive constant $M_{5}$ depending on $\varepsilon$ such that

$$
\begin{array}{r}
\int_{0}^{s} \int_{0}^{1} v_{y}^{2} d y d s \leq M_{5} \\
\int_{0}^{1} \theta_{y}^{2} d y \leq M_{5} .
\end{array}
$$

Proof. Multiplying (29) by $\rho_{0} v$ and using integration by parts, we have

$$
\begin{aligned}
& \frac{1}{2} \frac{d}{d s} \int_{0}^{1} \rho_{0} v^{2} d y+\varepsilon \int_{0}^{1} \frac{v_{y}^{2}}{1+\theta} d y+\int_{0}^{1} \frac{\rho_{0} v^{2}}{\tau_{p}} d y \\
= & \frac{e^{2}}{m} \int_{0}^{1} \rho_{0} v\left(\bar{\rho}_{0}-D\right) d y+\frac{1}{m} \int_{0}^{1} \frac{\rho_{0} v_{y}}{1+\theta} T d y .
\end{aligned}
$$

There exist positive constants $N_{1}$ and $N_{2}$ such that

$$
\begin{aligned}
\int_{0}^{1} v^{2} d y+\int_{0}^{s} \int_{0}^{1}\left(\varepsilon v_{y}^{2}+v^{2}\right) d y d s & \leq N_{1}+N_{2} \int_{0}^{s} \int_{0}^{1} T^{2} d y d s \\
& \leq N_{1}+N_{2} M_{4}
\end{aligned}
$$

by Lemma 4.

In order to prove (54), we rewrite (29) as follows:

$$
\frac{\varepsilon}{\rho_{0}}\left(\frac{\theta_{y}}{1+\theta}\right)_{s}+\frac{T}{m(1+\theta)}\left(\frac{\theta_{y}}{1+\theta}\right)=
$$




$$
=v_{s}+\frac{\left(\rho_{0} T\right)_{y}}{m \rho_{0}(1+\theta)}-\frac{e^{2}}{m}\left(\bar{\rho}_{0}-D\right)+\frac{v}{\tau_{p}} .
$$

Multiplying (55) by $\theta_{y} /(1+\theta)$ and integrating over $(0,1) \times(0, s)$, we have

$$
\begin{aligned}
& \frac{\varepsilon}{2} \int_{0}^{1} \frac{1}{\rho_{0}}\left(\frac{\theta_{y}}{1+\theta}\right)^{2} d y+\frac{1}{m} \int_{0}^{s} \int_{0}^{1} \frac{T \theta_{y}^{2}}{(1+\theta)^{3}} d y d s \int_{0}^{s} \int_{0}^{1}\left[\frac{\left(\rho_{0} T\right)_{y}}{m \rho_{0}(1+\theta)}-\frac{e^{2}}{m}\left(\bar{\rho}_{0}-D\right)+\frac{v}{\tau_{p}}\right] \frac{\theta_{y}}{1+\theta} d y d s \\
&+ \int_{0}^{s} \int_{0}^{1} v_{s}(\ln (1+\theta))_{y} d y d s \\
&=\int_{0}^{s} \int_{0}^{1}\left[\frac{\left(\rho_{0} T\right)_{y}}{m \rho_{0}(1+\theta)}-\frac{e^{2}}{m}\left(\bar{\rho}_{0}-D\right)+\frac{v}{\tau_{p}}\right] \frac{\theta_{y}}{1+\theta} d y d s+\int_{0}^{1} v \frac{\theta_{y}}{1+\theta} d y \\
&+\int_{0}^{s} \int_{0}^{1} \frac{v_{y}^{2}}{1+\theta} d y d s \\
& \leq \int_{0}^{s} \int_{0}^{1}\left[\frac{\left(\rho_{0} T\right)_{y}}{m \rho_{0}(1+\theta)}-\frac{e^{2}}{m}\left(\bar{\rho}_{0}-D\right)+\frac{v}{\tau_{p}}\right] \frac{\theta_{y}}{1+\theta} d y d s \\
&+\int_{0}^{s} \int_{0}^{1} \frac{v_{y}^{2}}{1+\theta} d y d s+\frac{\varepsilon}{2} \int_{0}^{1} \frac{1}{\rho_{0}}\left(\frac{\theta_{y}}{1+\theta}\right)^{2} d y+C(\varepsilon) \int_{0}^{1} \rho_{0} v^{2} d y .
\end{aligned}
$$

It follows from (56), Lemma 1, Lemma 3, and Lemma 4 that there exist two positive constants $N_{3}$ and $N_{4}$ depending on $\varepsilon$ such that

$$
\int_{0}^{1} \theta_{y}^{2} d y \leq N_{3}+N_{4} \int_{0}^{s} \int_{0}^{1} \theta_{y}^{2} d y d s .
$$

Use of Gronwall's inequality completes the proof of Lemma 5.

Lemma 6. There exist positive constants $M_{6}$ and $M_{7}$ depending on $\varepsilon$ such that

$$
\begin{aligned}
& \int_{0}^{1} v_{y}^{2} d y+\int_{0}^{s} \int_{0}^{1} v_{y y}^{2} d y d s \leq M_{6} \\
& \int_{0}^{1} T_{y}^{2} d y+\int_{0}^{s} \int_{0}^{1} T_{y y}^{2} d y d s \leq M_{7} .
\end{aligned}
$$


Proof. Multiplying (29) by $v_{y y}$ and integrating over $(0,1)$, we have

$$
\begin{aligned}
& \frac{1}{2} \frac{d}{d s} \int_{0}^{1} v_{y}^{2} d y+\varepsilon \int_{0}^{1} \frac{v_{y y}^{2}}{\rho_{0}(1+\theta)} d y \\
= & \varepsilon \int_{0}^{1} \frac{\theta_{y} v_{y} v_{y y}}{\rho_{0}(1+\theta)^{2}} d y+\frac{1}{m} \int_{0}^{1}\left(\frac{\left(\rho_{0} T\right)_{y}}{\rho_{0}(1+\theta)}-\frac{T \theta_{y}}{(1+\theta)^{2}}\right) v_{y y} d y \\
& -\int_{0}^{1}\left[\frac{e^{2}}{m}\left(\bar{\rho}_{0}-D\right)-\frac{v}{\tau_{p}}\right] v_{y y} d y .
\end{aligned}
$$

Using interpolation inequalities and Lemma 3, we obtain

$$
\begin{gathered}
\int_{0}^{1} v_{y}^{2} d y+\int_{0}^{s} \int_{0}^{1} v_{y y}^{2} d y d s \leq N_{1}+N_{2} \int_{0}^{s} \int_{0}^{1}\left(v_{y}^{2}+T^{2}\right) \theta_{y}^{2} d y d s \\
\leq N_{1}+N_{2} \int_{0}^{s} \int_{0}^{1}\left[\beta \int_{0}^{1} v_{y y}^{2} d y+C_{\beta} \int_{0}^{1} v_{y}^{2} d y\right. \\
\left.+2 \int_{0}^{1} T_{y}^{2} d y+2\left(\int_{0}^{1} T d y\right)^{2}\right] \theta_{y}^{2} d y d s .
\end{gathered}
$$

It follows from Lemma 4 and Lemma 5 that

$$
\begin{aligned}
\int_{0}^{1} v_{y}^{2} d y d s+\int_{0}^{s} \int_{0}^{1} v_{y y}^{2} d y d s & \leq 2 N_{1}+2 N_{2}\left(C_{\beta} M_{5}+2 M_{4}+2 M_{1}^{2}\right) M_{5} \\
& \equiv M_{6} .
\end{aligned}
$$

Multiplying (30) by $T_{y y}$ and integrating over $(0,1)$, we have

$$
\begin{aligned}
\frac{1}{2} \frac{d}{d s} \int_{0}^{1} T_{y}^{2} d y+\frac{3}{2} \int_{0}^{1} \frac{\kappa T_{y y}^{2}}{\rho_{0}(1+\theta)} d y & =\frac{2}{3} \int_{0}^{1}\left(\frac{v_{y} T}{1+\theta}-\frac{1}{\rho_{0}}\left(\frac{\kappa}{1+\theta}\right)_{y} T_{y}\right) T_{y y} d y \\
& +\int_{0}^{1}\left(\frac{T-T^{(0)}}{\tau_{w}}-\frac{m v^{2}\left(2 \tau_{w}-\tau_{p}\right)}{3 \tau_{p} \tau_{w}}\right) T_{y y} d y .
\end{aligned}
$$

Then, it follows from the interpolation inequalities and Lemma 3, Lemma 4, and Lemma 5 that 


$$
\begin{aligned}
& \int_{0}^{1} T_{y}^{2} d y+\int_{0}^{s} \int_{0}^{1} T_{y y}^{2} d y d s \\
\leq & N_{1}+N_{2} \int_{0}^{s} \int_{0}^{1}\left(T^{2} v_{y}^{2}+\theta_{y}^{2} T_{y}^{2}\right) d y d s \\
\leq & N_{1}+2 N_{2} \int_{0}^{s} \int_{0}^{1}\left(\int_{0}^{1} T_{y}^{2} d y+\left(\int_{0}^{1} T d y\right)^{2}\right) v_{y}^{2} d y d s \\
& +N_{2} \int_{0}^{s} \int_{0}^{1}\left(\beta \int_{0}^{1} T_{y y}^{2} d y+C_{\beta} \int_{0}^{1} T_{y}^{2} d y\right) \theta_{y}^{2} d y d s \\
\leq & N_{1}+2 N_{2} M_{6}\left(M_{4}+M_{1}^{2}\right)+C_{\beta} N_{2} M_{4} M_{5}+\beta N_{2} \int_{0}^{1} T_{y y}^{2} d y \\
\equiv & \frac{1}{2} M_{7}+\frac{1}{2} \int_{0}^{1} T_{y y}^{2} d y .
\end{aligned}
$$

This completes the proof of Lemma 6 .

Lemma 7. The velocity $v$ and temperature $T$ are bounded; that is,

$$
|v| \leq\left(M_{6}\right)^{1 / 2}, \quad 0<T \leq M_{1}+\left(M_{7}\right)^{1 / 2} .
$$

Proof. From Lemma 6, we have

$$
v^{2}(x, t)=\left(\int_{0}^{y} v_{y} d y\right)^{2} \leq \int_{0}^{1} v_{y}^{2} d y \leq M_{6} .
$$

Note that

$$
\left(T(y, s)-\int_{0}^{1} T(z, s) d z\right)^{2} \leq\left(\int_{0}^{1}\left|T_{y}\right| d y\right)^{2} \leq M_{7}
$$

by Lemma 6 . By Lemma 1 ,

$$
\int_{0}^{1} T(y, s) d y \leq M_{1}
$$


and the upper bound for $T$ follows.

From the a priori estimates above and the standard method applied in $[14,16]$, we obtain the following Schauder estimates.

Lemma 8. There exists a positive constant $M_{8}$ depending on $\varepsilon$ such that

$$
\|v\|_{H_{\alpha}(Q)}+\|T\|_{H_{\alpha}(Q)} \leq M_{8}, \quad 0<\alpha<1 .
$$

We omit the proof of Lemma 8. For details, see Theorem 2 of [16].

Lemma 9. There exists a positive constant $M_{9}$ such that

$$
T(y, s) \geq M_{9}>0, \quad(y, s) \in \bar{Q} .
$$

Proof. Let $\omega=T^{-1}$ and multiply (30) by $-\omega^{2}$; we have

$$
\begin{aligned}
\omega_{s}= & \frac{2}{3 \rho_{0}}\left(\frac{\kappa \omega_{y}}{1+\theta}\right)_{y}+\left(\frac{1}{\tau_{w}}+\frac{2 v_{y}}{3(1+\theta)}\right) \omega \\
& -\left[\frac{4 \kappa \omega_{y}^{2}}{3 \rho_{0} \omega^{3}(1+\theta)}+\frac{m v^{2}\left(2 \tau_{w}-\tau_{p}\right)}{3 \tau_{p} \tau_{w}}+\frac{T^{(0)}}{\tau_{w}}\right] \omega^{2} \\
\leq & \frac{2}{3 \rho_{0}}\left(\frac{\kappa \omega_{y}}{1+\theta}\right)_{y}+\left(\frac{1}{\tau_{w}}+\frac{2 v_{y}}{3(1+\theta)}\right) \omega .
\end{aligned}
$$

For $\ell=1,2,3, \ldots$, multiply (57) by $2 \ell \omega^{2 \ell-1}$ to obtain

$$
\begin{array}{r}
\left(\omega^{2 \ell}\right)_{s} \leq 2 \ell\left(\frac{1}{\tau_{w}}+\frac{2 v_{y}}{3(1+\theta)}\right) \omega^{2 \ell}+\frac{2}{3 \rho_{0}}\left(\frac{\kappa\left(\omega^{2 \ell}\right)_{y}}{1+\theta}\right)_{y} \\
-4 \ell(2 \ell-1) \frac{\kappa \omega_{y}^{2}}{3 \rho_{0}(1+\theta)} \omega^{2(\ell-1)} \\
\leq 2 \ell\left(\frac{1}{\tau_{w}}+\frac{2 v_{y}}{3(1+\theta)}\right) \omega^{2 \ell}+\frac{2}{3 \rho_{0}}\left(\frac{\kappa\left(\omega^{2 \ell}\right)_{y}}{1+\theta}\right)_{y} .
\end{array}
$$

Therefore,

$$
2 \ell\left\|\rho_{0}^{1 /(2 \ell)} \omega\right\|_{L^{2 \ell}}^{2 \ell-1} \frac{d}{d s}\left\|\rho_{0}^{1 /(2 \ell)} \omega\right\|_{L^{2 \ell}}(s) \leq 2 \ell \int_{0}^{1} \rho_{0}\left(\frac{1}{\tau_{w}}+\frac{2 v_{y}}{3(1+\theta)}\right) \omega^{2 \ell} d y ;
$$


that is,

$$
\frac{d}{d s}\left\|\rho_{0}^{1 /(2 \ell)} \omega\right\|_{L^{2 \ell}}(s) \leq\left(\delta^{-1}+M_{8} M_{3}\right)\left\|\rho_{0}^{1 /(2 \ell)} \omega\right\|_{L^{2 \ell}}(s) .
$$

It follows from Gronwall's inequality that

$$
\left\|\rho_{0}^{1 /(2 \ell)} \omega\right\|_{L^{2 \ell}}(s) \leq\left\|\rho_{0}^{1 /(2 \ell)} \omega_{0}\right\|_{L^{2 \ell}} e^{\left(\delta^{-1}+M_{3} M_{8}\right) S} .
$$

Letting $\ell \rightarrow \infty$, we obtain

$$
\frac{1}{\min T(y, s)} \leq \frac{1}{\delta} e^{\left(\delta^{-1} \delta+M_{3} M_{8}\right) S} .
$$

Thus,

$$
\min T(y, s) \geq \delta e^{-\left(\delta^{-1}+M_{3} M_{8}\right) S} \equiv M_{9}>0 .
$$

This completes the proof of Lemma 8 .

4. Existence and uniqueness. In this section we apply the Leray-Schauder fixed point theorem to the integro-differential equations (29)-(30). First, we define a set

$$
\begin{array}{r}
\mathbf{F}=\left\{(\tilde{v}, \tilde{T}): \frac{1}{M_{3}} \leq 1+\tilde{\theta} \leq M_{3},|\tilde{v}| \leq M_{6}, 0 \leq \tilde{T} \leq M_{1}+\left(M_{7}\right)^{1 / 2}\right. \\
\left.\|\tilde{v}, \tilde{T}\|_{H_{\alpha}(\bar{Q})} \leq M_{8}, \tilde{v}(y, 0)=v_{0}(y), \tilde{T}(y, 0)=T_{0}(y)\right\},
\end{array}
$$

where

$$
\tilde{\theta}=\int_{0}^{s} \tilde{v}_{y} d \sigma
$$

and constants $M_{i}$ have been determined in the previous section. It is clear that $\mathbf{F}$ is a closed subset of the Banach space $H_{\alpha}(\bar{Q})$.

We define an operator

$$
\Sigma:(\tilde{v}, \tilde{T}, \mu) \mapsto(v, T)
$$

by solving the following linear integro-differential equations: 


$$
\left\{\begin{aligned}
& v_{s}-\frac{\varepsilon}{\rho_{0}(1+\tilde{\theta})} v_{y y}+\frac{v}{\tilde{\tau}_{p}}=\frac{\mu\left(\rho_{0} \tilde{T}-m \varepsilon\right)}{m \rho_{0}(1+\tilde{\theta})^{2}} \theta\left(v_{y}\right) \\
&+\mu\left[\frac{e^{2}}{m}\left(\tilde{v} \rho_{0}-D\right)-\frac{\left(\rho_{0} \tilde{T}\right)_{y}}{m \rho_{0}(1+\tilde{\theta})}\right], \\
& T_{s}-\frac{2 \tilde{\kappa}}{3 \rho_{0}(1+\tilde{\theta})} T_{y y}+\frac{T-\mu T^{(0)}}{\tilde{\tau}_{w}}=-\frac{2 \mu \tilde{T}_{y}\left(\rho_{0} \tilde{\kappa}^{\prime}+\tilde{\kappa}(1+\tilde{\theta})\right)}{3 \rho_{0}(1+\tilde{\theta})^{3}} \theta\left(v_{y}\right) \\
&+\mu {\left[\frac{2 \rho_{0}^{\prime} \tilde{T}_{y} \tilde{\kappa}^{\prime}}{3 \rho_{0}(1+\tilde{\theta})^{2}}-\frac{2 \tilde{T} \tilde{v}_{y}}{3(1+\tilde{\theta})}+\frac{m \tilde{v}^{2}\left(2 \tilde{\tau}_{w}-\tilde{\tau}_{p}\right)}{3 \tilde{\tau}_{p} \tilde{\tau}_{w}}\right], }
\end{aligned}\right.
$$

with the following initial-boundary conditions:

$$
\begin{gathered}
v(y, 0)=\mu v_{0}(y), \quad T(y, 0)=\mu T_{0}(y), \\
v(0, s)=v(1, s)=0, \quad T_{y}(0, s)=T_{y}(1, s)=0,
\end{gathered}
$$

where $\mu \in[0,1],(\tilde{v}, \tilde{T}) \in \mathbf{F}, \tilde{\kappa}=\kappa\left(\rho_{0} /(1+\tilde{\theta})\right), \tilde{\tau}_{p}=\tau_{p}\left(\rho_{0} /(1+\tilde{\theta}), \tilde{v}, \tilde{T}\right)$, and $\tilde{\tau}_{w}=\tau_{w}\left(\rho_{0} /(1+\tilde{\theta}), \tilde{v}, \tilde{T}\right)$.

We prove the following theorem to see that the linear operator $\Sigma$ is well defined from the closed subset $\mathbf{F}$ of $H_{\alpha}(\bar{Q})$ into $C^{2+\alpha, 1+\alpha / 2}(\bar{Q})$ for every fixed $\mu \in[0,1]$.

Theorem 3. Assume that $\left(\mathrm{A}_{1}\right)$ and $\left(\mathrm{A}_{2}\right)$ hold. Then, for an arbitrary time $S \in(0, \infty)$, there exists a unique solution $(v, T) \in C^{2+\alpha, 1+\alpha / 2}(\bar{Q})$ of $(60)$ satisfying the following estimate:

$$
\|v\|_{2+\alpha, \bar{Q}}+\|T\|_{2+\alpha, \bar{Q}} \leq \bar{M}\left(\left\|v_{0}\right\|_{w+\alpha}+\left\|T_{0}\right\|_{2+\alpha}+\|\tilde{v}\|_{2+\alpha, \bar{Q}}+\|\tilde{T}\|_{2+\alpha, \bar{Q}}\right),
$$

where constant $\bar{M}$ is independent of $(v, T)$.

Proof. For simplicity, we write the equations of (60) as follows:

$$
\left\{\begin{array}{l}
v_{s}-a_{1} v_{y y}+\frac{v}{\tilde{\tau}_{p}}=b_{1} \int_{0}^{s} v_{y y} d \sigma+f_{1}, \\
T_{s}-a_{2} T_{y y}+\frac{T-\mu T^{(0)}}{\tilde{\tau}_{w}}=b_{2} \int_{0}^{s} v_{y y} d \sigma+f_{2},
\end{array}\right.
$$


where

$$
\begin{aligned}
& a_{1}=\frac{\varepsilon}{\rho_{0}(1+\tilde{\theta})}, \quad a_{2}=\frac{2 \tilde{\kappa}}{3 \rho_{0}(1+\tilde{\theta})}, \\
& b_{1}=\frac{\mu\left(\rho_{0} \tilde{T}-m \varepsilon\right)}{m \rho_{0}(1+\tilde{\theta})^{2}}, \quad b_{2}=-\frac{2 \mu \tilde{T}_{y}\left(\rho_{0} \tilde{\kappa}^{1}+\tilde{\kappa}(1+\tilde{\theta})\right)}{3 \rho_{0}(1+\tilde{\theta})^{3}}, \\
& f_{1}=\mu\left[\frac{e^{2}}{m}\left(\tilde{v} \rho_{0}-D\right)-\frac{\left(\rho_{0} \tilde{T}\right)_{y}}{m \rho_{0}(1+\tilde{\theta})}\right] \\
& f_{2}=\mu\left[\frac{2 \rho_{0}^{\prime} \tilde{T}_{y} \tilde{\kappa}^{\prime}}{3 \rho_{0}(1+\tilde{\theta})^{2}}-\frac{2 \tilde{T} \tilde{v}_{y}}{3(1+\tilde{\theta})}+\frac{m \tilde{v}^{2}\left(2 \tilde{\tau}_{w}-\tilde{\tau}_{p}\right)}{3 \tilde{\tau}_{p} \tilde{\tau}_{w}}\right] .
\end{aligned}
$$

Notice that $a_{i}, b_{i}, f_{i}, i=1,2$, are Hölder continuous by assumptions on the given functions and $(\tilde{v}, \tilde{T})$.

Suppose that $(v, T) \in C^{2+\alpha, 1+\alpha / 2}$ satisfies (60). We can visualize the integrodifferential equations of (60) as a couple of parabolic equations [4] with two source terms:

$$
b_{i} \int_{0}^{s} v_{y y} d \sigma+f_{i}, \quad i=1,2
$$

Then, for every rectangle $Q_{s_{0}} \equiv(0,1) \times\left(0, s_{0}\right), s_{0} \in(0, S]$, the following estimate $[7,17]$ holds:

$$
\begin{array}{r}
\|v\|_{2+\alpha, \bar{Q}_{s_{0}}}+\|T\|_{2+\alpha, \bar{Q}_{s_{0}}} \leq \bar{M}_{1}\left(\left\|v_{0}\right\|_{2+\alpha}+\left\|T_{0}\right\|_{2+\alpha}+\sum_{i=1}^{2}\left|f_{i}\right|_{\alpha, \bar{Q}_{s_{0}}}\right. \\
\left.+\left|\int_{0}^{s} v_{y y} d \sigma\right|_{\alpha, \bar{Q}_{s_{0}}} \sum_{i=1}^{2}\left|b_{i}\right|_{\alpha, \bar{Q}_{s_{0}}}\right),
\end{array}
$$

where $\bar{M}_{1}$ is independent of $(v, T)$.

By assumption on $(\tilde{v}, \tilde{T})$, there exists a $s_{0}>0$ independent of $(v, T)$ such that

$$
\bar{M}_{1}\left(\sum_{i=1}^{2}\left|b_{i}\right|_{\alpha, \bar{Q}}\right)\left(s_{0}+s_{0}^{1-\alpha / 2}\right) \leq \frac{1}{2} .
$$


For such a $s_{0}>0$, it follows from (62) that

$$
\|v\|_{2+\alpha, \bar{Q}_{s_{0}}}+\|T\|_{2+\alpha, \bar{Q}_{s_{0}}} \leq 2 \bar{M}_{1}\left(\left\|v_{0}\right\|_{2+\alpha}+\left\|T_{0}\right\|_{2+\alpha}+\sum_{i=1}^{2}\left|f_{i}\right|_{\alpha, \bar{Q}_{s_{0}}}\right) .
$$

In particular,

$$
\left\|v\left(\cdot, s_{0}\right)\right\|_{2+\alpha}+\left\|T\left(\cdot, s_{0}\right)\right\|_{2+\alpha} \leq 2 \bar{M}_{1}\left(\left\|v_{0}\right\|_{2+\alpha}+\left\|T_{0}\right\|_{2+\alpha}+\sum_{i=1}^{2}\left|f_{i}\right|_{\alpha, \bar{Q}_{s_{0}}}\right) .
$$

Repeating the same procedure for the rectangles $(0,1) \times\left(k s_{0},(k+1) s_{0}\right)$, $k=1,2, \ldots, 1+\left[\frac{S}{s_{0}}\right]$, we have

$$
\begin{aligned}
& \|v\|_{2+\alpha, \bar{Q}}+\|T\|_{2+\alpha, \bar{Q}} \\
\leq & \left(1+2 \bar{M}_{1}\right)^{1+\left[S / s_{0}\right]}\left(\left\|v_{0}\right\|_{2+\alpha}+\left\|T_{0}\right\|_{2+\alpha}+\sum_{i=1}^{2}\left|f_{i}\right|_{\alpha, \bar{Q}}\right) .
\end{aligned}
$$

It follows from (63) that (61).

The uniqueness of the solution of (60) follows immediately from (63). To show the existence of the solution (60), we consider the one parameter family of operators for $\lambda \in[0,1]$ :

$$
\begin{aligned}
& L_{1, \lambda}(v):=v_{s}-\left(\lambda a_{1}+(1-\lambda) \frac{\varepsilon}{n_{0}}\right) v_{y y}+\lambda\left(\frac{v}{\tilde{\tau}_{p}}-b_{1} \int_{0}^{s} v_{y y} d \sigma\right) \\
& L_{2, \lambda}(T):=T_{s}-\left(\lambda a_{2}+(1-\lambda) \frac{2 \tilde{\kappa}}{3 n_{0}}\right) T_{y y}+\lambda\left(\frac{T-\mu T^{(0)}}{\tilde{\tau}_{w}}-b_{2} \int_{0}^{s} v_{y y} d \sigma\right) .
\end{aligned}
$$

Let $\Omega$ be the set of all $\lambda$ for which the problem

$$
\left\{\begin{array}{l}
L_{1, \lambda}(v)=f_{1}, \\
L_{2, \lambda}(T)=f_{2}, \\
v(y, 0)=\mu v_{0}(y), \quad T(y, 0)=\mu T_{0}(y), \quad y \in[0,1], \\
v(0, s)=v(1, s)=0, \\
T_{y}(0, s)=T_{y}(1, s)=0,
\end{array}\right.
$$

has a unique solution $(v, T) \in C^{2+\alpha, 1+\alpha / 2}(\bar{Q})$ for every couple of functions $\left(f_{1}, f_{2}\right) \in C^{\alpha, \alpha / 2}(\bar{Q})$.

It is easy to check that the compatibility conditions of problem (64) hold for $\lambda=0$. By the results of the standard parabolic equations, we have $0 \in \Omega$. eject

To this end, we show that $\Omega$ is both open and closed. Suppose $\lambda_{0} \in \Omega$. Given 
$(v, T) \in C^{2+\alpha, 1+\alpha / 2}(\bar{Q})$, we define a linear operator $\left(\psi_{1}, \psi_{1}\right)=H_{\lambda}(v, T)$ to be the unique solution of the following problem:

$$
\left\{\begin{array}{l}
L_{1, \lambda_{0}}\left(\psi_{1}\right)=L_{1, \lambda_{0}}(v)-L_{1, \lambda}(v)+f_{1}, \\
L_{2, \lambda_{0}}\left(\psi_{2}\right)=L_{2, \lambda_{0}}(t)-L_{2, \lambda}(T)+f_{2}, \\
\psi_{1}(y, 0)=\mu v_{0}(y), \quad \psi_{2}(y, 0)=\mu T_{0}(y), \quad y \in[0,1], \\
\psi_{1}(0, s)=\psi_{1}(1, s)=0, \\
\psi_{2, y}(0, s)=\psi_{2, y}(1, s)=0 .
\end{array}\right.
$$

The operator $\left(\psi_{1}, \psi_{2}\right)=H_{\lambda}(v, T)$ is well defined since

$$
L_{1, \lambda_{0}}(v)-L_{1, \lambda}(v)+f_{1}, L_{2, \lambda_{0}}(T)-L_{2, \lambda}(T)+f_{2} \in C^{\alpha, \alpha / 2}(\bar{Q}) .
$$

Notice that (65) is of the form (60). As in (63), there exists a constant $\bar{M}_{2}$ independent of $\left(\psi_{1}, \psi_{2}\right)$ such that

$$
\begin{aligned}
& \left\|\psi_{1}\right\|_{2+\alpha, Q}+\left\|\psi_{2}\right\|_{2+\alpha, Q} \\
\leq & \bar{M}_{2}\left\{\left\|v_{0}\right\|_{2+\alpha}+\left\|T_{0}\right\|_{2+\alpha}+\sum_{i=1}^{2}\left|f_{i}\right|_{\alpha, \bar{Q}}+\left|\lambda-\lambda_{0}\right|\left(\|v\|_{2+\alpha, \bar{Q}}+\|T\|_{2+\alpha, \bar{Q}}\right)\right\} .
\end{aligned}
$$

We consider the following closed subset $X$ of $C^{2+\alpha, 1+\alpha / 2}(\bar{Q})$ :

$$
X:=\left\{(v, T):\|v, T\|_{2+\alpha, \bar{Q}} \leq 2 \bar{M}_{2}\left(\left\|v_{0}\right\|_{2+\alpha}+\left\|T_{0}\right\|_{2+\alpha}+\sum_{i=1}^{2}\left|f_{i}\right|_{\alpha, \bar{Q}}\right)\right\} .
$$

Then $H_{\lambda}$ maps $X$ into itself if $\left|\lambda-\lambda_{0}\right|<1\left(2 \bar{M}_{2}\right)$. Furthermore, if $\left(v_{1}, T_{1}\right)$, $\left(v_{2}, T_{2}\right) \in X$, then

$$
\left.\left\|H_{\lambda}\left(v_{2}, T_{2}\right)-H_{\lambda}\left(v_{1}, T_{1}\right)\right\|_{2+\alpha, \bar{Q}} \leq \frac{1}{2}\left\|v_{2}-v_{1}\right\|_{2+\alpha, \bar{Q}}+\left\|T_{2}-T_{1}\right\|_{2+\alpha, \bar{Q}}\right) .
$$

It follows from the contraction principle that there exists a unique fixed point $(v, T)=H_{\lambda}(v, T)$ in $X$. Thus, $\lambda \in \Omega$ and $\Omega$ is open.

Suppose $\lambda \in \bar{\Omega}$. Then there exists a sequence $\lambda_{\ell} \subset \Omega$ such that $\lambda_{\ell} \rightarrow \lambda$ as $\ell \rightarrow \infty$. By assumption, the equation (64) has a unique solution $\left(v_{\ell}, T_{\ell}\right) \in$ $C^{2+\alpha, 1+\alpha / 2}(\bar{Q})$ for every $\lambda_{\ell}$. As in (63), we can prove that $\left\{\left(v_{\ell}, T_{\ell}\right)\right\}$ is a Cauchy sequence in $C^{2+\alpha, 1+\alpha / 2}(\bar{Q})$. Then there exists a unique $(v, T)$ such that $\left(v_{\ell}, T_{\ell}\right) \rightarrow(v, T)$ in $C^{2+\alpha, 1+\alpha / 2}(\bar{Q})$ as $\ell \rightarrow \infty$. Letting $\ell \rightarrow \infty$ in (64), we have that $(v, T)$ is a unique solution of (64) corresponding to $\lambda$. Thus, $\lambda \in \Omega$ and $\Omega$ is closed. Therefore, $1 \in \Omega$. 
This completes the proof of Theorem 3 .

4.1. Proof of Theorem 1. For the set $\mathbf{F}$ and mapping $(v, T)=\Sigma(\tilde{v}, \tilde{T}, \mu)$ defined by (59) and (60), we can easily verify all conditions required in the Leray-Schauder fixed point theorem by a standard argument. Thus, there exists at least a fixed point $(v, T)$ of $\Sigma(\cdot, \cdot, 1)$ in $\mathbf{F}$. We omit the details of verification of conditions for the Leray-Schauder fixed point theorem. From the regularity property of the solution of linear parabolic equations, we conclude that $(v, T) \in$ $C^{2+\alpha, 1+\alpha / 2}(\bar{Q})$. By the equivalence of $(1)-(7)$ and $(29)-(32)$, there exists a solution $(\rho, v, T, \varphi)$ of $(1)-(7)$, where the electron density $\rho$ and electrostatic potential $\varphi$ are defined by (24) and (25), respectively.

Now we show the uniqueness. Suppose that there are two solutions

$$
\left(\rho_{i}, v_{i}, T_{i}, \varphi_{i}\right), \quad i=1,2 \text {. }
$$

Let

$$
(\rho, v, T, \varphi)=\left(\rho_{2}-\rho_{1}, v_{2}-v_{1}, T_{2}-T_{1}, \varphi_{2}-\varphi_{1}\right)
$$

Then $(\rho, v, T, \varphi)$ satisfies the following initial-boundary value problem:

$$
\begin{aligned}
& \rho_{2} v_{t}-\varepsilon v_{x x}+\frac{1}{2} \rho_{2}\left(\left(v_{2}+v_{1}\right) v\right)_{x}+\frac{1}{m}\left(\rho_{2} T+T_{1} \rho\right)_{x}+\left(v_{1 t}+\frac{1}{2}\left(v_{1}^{2}\right)_{x}\right) \rho \\
= & \frac{e}{m}\left(\rho_{2} \varphi_{x}+\varphi_{1} \rho\right)-\frac{\left(v_{2} \rho-\rho_{1} v\right) \tau_{p}^{(1)}-\rho_{1} v_{1}\left(\tau_{p}^{(2)}-\tau_{p}^{(1)}\right)}{\tau_{p}^{(1)} \tau_{p}^{(2)}}
\end{aligned}
$$

$$
\begin{aligned}
\rho_{2} T_{t}- & \frac{2}{3}\left(\kappa_{2} T_{x}\right)_{x}-\frac{2}{3}\left(\left(\kappa_{2}-\kappa_{1}\right) T_{1 x}\right)_{x} \\
& +T_{1 t} \rho+\left(\rho_{2} v_{2}\right) T_{x}+\left(\rho_{2} T_{1 x}\right) v+\left(v_{1} T_{1 x}\right) \rho \\
& +\frac{2}{3}\left(\left(\rho_{2} T_{2}\right) v_{x}+\left(\rho_{2} v_{1 x}\right) T+\left(T_{1} v_{1 x}\right) \rho\right) \\
=\frac{m v_{2}^{2}+}{3\left(T_{1}+T^{(0)}\right)} & \left.3 \tau_{w}^{(1)} \tau_{w}^{(2)}(2)-\tau_{w}^{(1)}\right) \\
& +\frac{2 m v_{1}^{2}}{\tau_{p}^{(1)} \tau_{p}^{(2)}}\left(\tau_{p}^{(2)}-\tau_{p}^{(1)}\right)+\frac{m\left(v_{2}+v_{1}\right)\left(2 \tau_{w}^{(1)}-\tau_{p}^{(1)}\right)}{3 \tau_{p}^{(2)} \tau_{2}^{(1)}} v-\frac{T}{\tau_{w}^{(2)}},
\end{aligned}
$$

(69) $\varphi_{x x}=e \rho$, 


$$
\begin{aligned}
& \rho(x, 0)=v(x, 0)=T(x, 0)=0, \quad x \in[0,1] \\
& v(0, t)=v(1, t)=T_{x}(0, t)=T_{x}(1, t)=0, \\
& \varphi_{x}(0, t)=\varphi(1, x)=0, \quad t \geq 0,
\end{aligned}
$$

where $\kappa_{i}=\kappa\left(\rho_{i}\right), \tau_{p}^{(i)}=\tau_{p}\left(\rho_{i}, v_{i}, T_{i}\right)$, and $\tau_{w}^{(i)}=\tau_{w}\left(\rho_{i}, v_{i}, T_{i}\right), i=1,2$.

Multiplying (66), (67), and (68) by $\rho, v$, and $T$, respectively, we have

$$
\begin{aligned}
& \frac{1}{2} \frac{d}{d t} \int_{0}^{1} \rho^{2} d x=-\int_{0}^{1}\left(\frac{1}{2} v_{2 x} \rho^{2}+\left(\rho_{1} v\right)_{x} \rho\right) d x \\
\leq & \frac{\varepsilon}{6} \int_{0}^{1} v_{x}^{2} d x+N_{1} \int_{0}^{1}\left(\rho^{2}+\rho_{2} v^{2}\right) d x \\
& \frac{1}{2} \frac{d}{d t} \int_{0}^{1} \rho_{2} v^{2} d x+\varepsilon \int_{0}^{1} v_{x}^{2} d x \\
\leq & \frac{\varepsilon}{6} \int_{0}^{1} v_{x}^{2} d x+N_{2} \int_{0}^{1}\left(\rho^{2}+\rho_{2}\left(v^{2}+T^{2}\right)\right) d x, \\
& \frac{1}{2} \frac{d}{d t} \int_{0}^{1} \rho_{2} T^{2} d x+\frac{2}{3} \int_{0}^{1} \kappa_{2} T_{x}^{2} d x \\
\leq & \frac{1}{3} \int_{0}^{1}\left(\kappa_{2} T_{x}^{2}+\frac{\varepsilon}{2} v_{x}^{2}\right) d x+N_{3} \int_{0}^{1}\left(\rho^{2}+\rho_{0}\left(v^{2}+T^{2}\right)\right) d x .
\end{aligned}
$$

where $N_{i}, i=1,2,3$, are some positive constants depending on $M_{k}, k=1, \ldots, 8$.

By (72)-(74), we have

$$
\frac{d}{d t} \int_{0}^{1}\left[\rho^{2}+\rho_{2}\left(v^{2}+T^{2}\right)\right] d x+\int_{0}^{1}\left(\varepsilon v_{x}^{2}+\frac{2 \kappa_{2}}{3} T_{x}^{2}\right) d x \leq N \int_{0}^{1}\left[\rho^{2}+\rho_{2}\left(v^{2}+T^{2}\right)\right] d x .
$$

It follows from Gronwall's inequality and (70)-(71) that

$$
\rho=v=T \equiv 0 .
$$

And $\varphi \equiv 0$ follows easily from (69) and (71). This completes the proof of Theorem 1 .

5. Asymptotic stability. In the previous section we proved that there exists a unique smooth solution of the initial-boundary problem (1)-(7) for an arbitrary time $S \in(0, \infty)$. Now we can investigate the asymptotic behavior of the 
solution of the problem (1)-(7) for the special data $\rho_{0}(x)=C(x), x \in[0,1]$. We first consider the following one-dimensional steady-state hydrodynamic equations with small viscosity:

$$
\begin{aligned}
& (\rho v)^{\prime}=0 \\
& \varepsilon v^{\prime \prime}=\rho v v^{\prime}+\frac{1}{m}(\rho T)^{\prime}-\frac{e}{m} \rho \varphi^{\prime}+\frac{\rho v}{\tau_{p}} \\
& \left(\kappa T^{\prime}\right)^{\prime}=\frac{3}{2} \rho v T^{\prime}+\rho T v^{\prime}-\frac{m \rho v^{2}\left(2 \tau_{w}-\tau_{p}\right)}{2 \tau_{p} \tau_{w}}+\frac{3 \rho\left(T-T^{(0)}\right)}{2 \tau_{w}}, \\
& \varphi^{\prime \prime}=e(\rho-C(x)), \\
& v(0)=v(1)=0, \quad T^{\prime}(0)=T^{\prime}(1)=0 \\
& \varphi^{\prime}(0)=0, \quad \varphi(1)=\varphi_{1},
\end{aligned}
$$

where $(\cdot)^{\prime}=\frac{d}{d x}(\cdot)$.

We also assume that

$$
\int_{0}^{1} \rho(x) d x=\int_{0}^{1} C(x) d x .
$$

Theorem 4. There exists a solution

$$
=\left(\frac{\int_{0}^{1} C(x) d x}{\int_{0}^{1} \exp \left(\frac{e\left(\varphi(x)-\varphi_{1}\right)}{T^{(0)}}\right) d x} \exp \left(\frac{e\left(\varphi(x)-\varphi_{1}\right)}{T^{(0)}}\right), 0, T^{(0)}, \varphi(x)\right)
$$

of the boundary value problem (75)-(80), where $\varphi(x)$ is a solution by solving

$$
\left\{\begin{array}{l}
\frac{1}{e} \varphi^{\prime \prime}(x)=\frac{\int_{0}^{1} C(x) d x}{\int_{0}^{1} \exp \left(\frac{e\left(\varphi(x)-\varphi_{1}\right)}{T^{(0)}}\right) d x} \exp \left(\frac{e\left(\varphi(x)-\varphi_{1}\right)}{T^{(0)}}\right)-C(x), \\
\varphi^{\prime}(0)=0, \quad \varphi(1)=\varphi_{1} .
\end{array}\right.
$$

Proof. From (75) and the boundary data (79), we have

$$
(\rho v)(x)=0, x \in[0,1] .
$$


Suppose that $\rho(x)>0$ for $x \in[0,1]$, then

$$
v(x)=0, x \in[0,1] \text {. }
$$

The equation (77) then reads:

$$
\left(\kappa T^{\prime}\right)^{\prime}=\frac{3 \rho\left(T-T^{(0)}\right)}{2 \tau_{w}} .
$$

Multiplying (82) by $T-T^{(0)}$ and using integration by parts, we obtain

$$
\int_{0}^{1} \kappa\left(T^{\prime}\right)^{2} d x+\int_{0}^{1} \frac{3 \rho\left(T-T^{(0)}\right)^{2}}{2 \tau_{w}} d x=0 .
$$

It follows that

$$
T(x)=T^{(0)}, \quad x \in[0,1] .
$$

The equation (76) then reads:

$$
\rho^{\prime}=\frac{e}{T^{(0)}} \rho \varphi^{\prime} .
$$

The unique solution of (83) is given by

$$
\rho(x)=\rho(1) \exp \left(\frac{e\left(\varphi(x)-\varphi_{1}\right)}{T^{(0)}}\right) .
$$

We claim that $\rho(1)$ is a positive constant depending only on $\varphi(x)$. By (78)(80) and assumption on $\rho(x)$, we have

$$
\varphi^{\prime}(1)=\varphi^{\prime}(0)+e\left(\int_{0}^{1} \rho(x) d x-\int_{0}^{1} C(x) d x\right)=0 .
$$

Substituting the expression (84) for $\rho(x)$ in (78) and integrating over $(0,1)$, we have

$$
\rho(1) \int_{0}^{1} \exp \left(\frac{e\left(\varphi(x)-\varphi_{1}\right)}{T^{(0)}}\right) d x-\int_{0}^{1} C(x) d x=\varphi^{\prime}(1)-\varphi^{\prime}(0)=0 .
$$

Thus,

$$
\rho(1)=\frac{\int_{0}^{1} C(x) d x}{\int_{0}^{1} \exp \left(\frac{e\left(\varphi(x)-\varphi_{1}\right)}{T^{(0)}} d x\right)}>0 .
$$


To this end, we have to show the existence of a solution of the following boundary value problem:

$$
\left\{\begin{array}{l}
\frac{1}{e} \varphi^{\prime \prime}(x)=\frac{\int_{0}^{1} C(x) d x}{\int_{0}^{1} \exp \left(\frac{e\left(\varphi(x)-\varphi_{1}\right)}{T^{(0)}}\right) d x} \exp \left(\frac{e\left(\varphi(x)-\varphi_{1}\right)}{T^{(0)}}\right)-C(x), \\
\varphi^{\prime}(0)=0, \quad \varphi(1)=\varphi_{1}
\end{array}\right.
$$

For a special case $C(x)=C=$ const. $>0$, there exists a trivial solution $\varphi=\varphi_{1}$ of $(85)$. Then $(\rho, v, T, \varphi)=\left(C, 0, T^{(0)}, \varphi_{1}\right)$ is a trivial solution of $(1)-(7)$, or $(75)-(80)$.

Using the boundary condition, we have

$$
\begin{aligned}
(86)\left|\varphi^{\prime}(x)\right|= & e\left|\frac{\int_{0}^{x} \exp \left(\frac{e\left(\varphi(x)-\varphi_{1}\right)}{\left.T^{(0)}\right)}\right) d x}{\int_{0}^{1} \exp \left(\frac{e\left(\varphi(x)-\varphi_{1}\right)}{T^{(0)}}\right) d x} \int_{0}^{1} C(x) d x-\int_{0}^{x} C(x) d x\right| \\
\leq & 2 e \int_{0}^{1} C(x) d x \equiv C_{1}, \\
(87) \quad & |\varphi(x)|=\left|\int_{1}^{x} \varphi^{\prime}(x) d x+\varphi_{1}\right| \leq C_{1}+\left|\varphi_{1}\right| \equiv C_{2} .
\end{aligned}
$$

We then define the following bounded, closed, and convex subset of $C^{0}[0,1]$ :

$$
X:=\left\{\psi(x):\left|\psi^{\prime}\right| \leq C_{1},|\psi| \leq C_{2}, \psi^{\prime}(0)=0, \psi(1)=\varphi_{1}\right\}
$$

We also consider the operator $\Sigma: \psi \rightarrow \varphi$ defined by solving

$$
(88)\left\{\begin{array}{l}
\frac{1}{e} \varphi^{\prime \prime}(x)=\frac{\int_{0}^{1} C(x) d x}{\int_{0}^{1} \exp \left(\frac{e\left(\psi(x)-\varphi_{1}\right)}{T^{(0)}}\right) d x} \exp \left(\frac{e\left(\psi(x)-\varphi_{1}\right)}{T^{(0)}}\right)-C(x), \\
\varphi^{\prime}(0)=0, \quad \varphi(1)=\varphi_{1},
\end{array}\right.
$$

where $\psi(x) \in X$.

The linear equation (88) is uniformly elliptic. Thus, the smoothness assumption on $C(x)$ guarantees the existence of a unique solution $\varphi=\Sigma(\psi)$ in $C^{2}[0,1]$. 
It is easy to check that the solution $\varphi=\Sigma(\psi)$ also satisfies (86)-(87); that is, $\varphi=\Sigma(\psi) \in X$. Thus, $\{\Sigma(\psi)\}$ is compact in $C^{0}[0,1]$ by the Ascoli-Arzela theorem. By a standard continuity argument, we conclude the existence of a fixed point of $\Sigma$ from the Schauder fixed point theorem. This completes the proof of Theorem 4.

5.1. Proof of Theorem 2. Multiplying (2) by $\rho v$ and using integration by parts, we have

(89) $\int_{0}^{1} \rho v^{2} d x+2 \int_{0}^{t} \int_{0}^{1}\left(\varepsilon v_{x}^{2}+\frac{\rho v^{2}}{\tau_{p}}\right) d x d t+\frac{2}{m}\left(I_{1}-e I_{2}\right)=\int_{0}^{1} \rho_{0} v_{0}^{2} d x$,

where

$$
\begin{aligned}
I_{1} & =\int_{0}^{t} \int_{0}^{1} v(\rho T)_{x} d x d t \\
I_{2} & =\int_{0}^{t} \int_{0}^{1} \rho v \varphi_{x} d x d t
\end{aligned}
$$

For $I_{1}$, we have

$$
\begin{aligned}
I_{1} & =-\int_{0}^{t} \int_{0}^{1} T\left(\rho v_{x}\right) d x d t \\
& =\int_{0}^{t} \int_{0}^{1} T\left(\rho_{t}+v \rho_{x}\right) d x d t \quad(\text { by }(1)) \\
& =\int_{0}^{t} \int_{0}^{1}(\rho T)_{t} d x d t+\int_{0}^{t} \int_{0}^{1}\left(T v \rho_{x}-\rho T_{t}\right) d x d t \\
& =\int_{0}^{t} \int_{0}^{1}\left(v(\rho T)_{x}+\frac{2}{3} \rho T v_{x}-\frac{2}{3}\left(\kappa T_{x}\right)_{x}-\frac{m \rho v^{2}\left(2 \tau_{w}-\tau_{p}\right)}{3 \tau_{p} \tau_{w}}\right. \\
& =\frac{1}{3} I_{1}+\int_{0}^{t} \int_{0}^{1}\left(\frac{\rho\left(T-T^{(0)}\right)}{\tau_{w}}\right) d x d t+\int_{0}^{1}\left(n T-n_{0} T_{0}\right) d x \\
& +\int_{0}^{1}\left(\rho T-\rho_{0} T_{0}\right) d x . \\
&
\end{aligned}
$$


Thus,

$$
\begin{aligned}
\frac{2}{m} I_{1}= & \int_{0}^{t} \int_{0}^{1}\left(\frac{3 \rho\left(T-T^{(0)}\right)}{m \tau_{w}}+\frac{\rho v^{2}}{\tau_{w}}\right) d x d t \\
& -2 \int_{0}^{t} \int_{0}^{1} \frac{\rho v^{2}}{\tau_{p}} d x d t+\frac{3}{m} \int_{0}^{1}\left(\rho T-\rho_{0} T_{0}\right) d x .
\end{aligned}
$$

For $I_{2}$, starting from (1), we have

$$
\int_{0}^{x} \rho d x=\int_{0}^{x} \rho_{0} d x-\int_{0}^{t} \rho v d t
$$

Thus,

$$
\varphi_{x}=\int_{0}^{x}(\rho-C(x)) d x=-\int_{0}^{t} \rho v d x \quad\left(\text { by } \rho_{0}(x)=C(x)\right) .
$$

It follows that

$$
\begin{aligned}
I_{2} & =-\int_{0}^{t} \int_{0}^{1}(\rho v)\left(\int_{0}^{t} \rho v d t\right) d x d t-\frac{1}{2} \int_{0}^{1}\left(\int_{0}^{t} \rho v d t\right)^{2} d x \\
& \equiv-\frac{1}{2} f(t) .
\end{aligned}
$$

Substituting (90) and (92) for $I_{1}$ and $I_{2}$ in (89), we obtain

$$
\begin{aligned}
& \int_{0}^{1}\left(\frac{3 \rho T}{m}+\rho v^{2}\right) d x+\frac{e}{m} f(t) \\
& +\int_{0}^{t} \int_{0}^{1}\left(2 \varepsilon v_{x}^{2}+\frac{m \rho v^{2}+3 \rho\left(T-T^{(0)}\right)}{m \tau_{w}}\right) d x d t \\
= & \int_{0}^{1} \rho_{0}\left(\frac{3 T_{0}}{m}+v_{0}^{2}\right) d x .
\end{aligned}
$$

It follows from (93) that

$$
\begin{aligned}
& \int_{0}^{1} \rho v^{2} d x \in L^{\infty}(0, \infty) \cap L^{1}(0, \infty), \\
& \int_{0}^{1} v_{x}^{2} d x \in L^{1}(0, \infty), \\
& f(t)=\int_{0}^{1}\left(\int_{0}^{\infty} \rho v d t\right)^{2} d x \in L^{\infty}(0, \infty) .
\end{aligned}
$$


Multiplying (3) by $\rho / T$ and using integration by parts, we have the following expression from the left-hand side:

$$
\begin{aligned}
\text { L.H.S. } & =\int_{0}^{1} \rho(\ln T)_{t} d x+\int_{0}^{1} \rho v(\ln T)_{x} d x+\frac{2}{3} \int_{0}^{1}\left(\rho v_{x}-\frac{1}{T}\left(\kappa T_{x}\right)_{x}\right) d x \\
& =\frac{d}{d t} \int_{0}^{1} \rho \ln T d x+\int_{0}^{1}\left(\rho v(\ln T)_{x}-(\ln T) \rho_{t}\right) d x+\frac{2}{3} \int_{0}^{1} \rho v_{x} d x \\
& =\frac{d}{d t} \int_{0}^{1} \rho \ln T d x+\frac{2}{3} \int_{0}^{1} \rho v_{x} d x-\frac{2}{3} \int_{0}^{1} \kappa\left(\frac{T_{x}}{T}\right)^{2} d x
\end{aligned}
$$

Thus,

$$
\begin{aligned}
& \int_{0}^{1} \rho \ln T d x+\int_{0}^{t} \int_{0}^{1}\left[\frac{\rho\left(T-T^{(0)}\right)}{T \tau_{w}} \frac{m \rho v^{2}\left(2 \tau_{w}-\tau_{p}\right)}{3 T \tau_{p} \tau_{w}}-\frac{2 \kappa}{3}\left(\frac{T_{x}}{T}\right)^{2}\right] d x d t \\
= & \int_{0}^{1} \rho_{0} \ln T_{0} d x-\frac{2}{3} \int_{0}^{t} \int_{0}^{1} \rho v_{x} d x .
\end{aligned}
$$

On the other hand, we multiply (3) by $1 / T$ and use integration by parts to obtain

$$
\begin{aligned}
\text { L. H.S. }= & \frac{3}{2} \int_{0}^{1}\left(\rho(\ln \rho)_{t}+\rho(\ln T)_{t}\right) d x+\frac{m}{2} \int_{0}^{1}\left(\frac{v}{T}(\rho v)_{t}+\frac{\rho v}{T} v_{t}\right) d x \\
& +\int_{0}^{1} \frac{1}{T}\left(\frac{5}{2} \rho v T+\frac{1}{2} m \rho v^{3}\right)_{x} d x \\
& -\int_{0}^{1} \kappa\left(\frac{T_{x}}{T}\right)^{2} d x-\varepsilon m \int_{0}^{1} \frac{v v_{x x}}{T} d x \\
= & \frac{3}{2} \frac{d}{d t} \int_{0}^{1} \rho(\ln \rho+\ln T) d x-\frac{3}{2} \int_{0}^{1}(\ln \rho+\ln T) \rho_{t} d x \\
& -\frac{3}{2} \int_{0}^{1}(\ln T)(\rho v)_{x} d x+\int_{0}^{1} \rho v_{x} d x \\
& -\int_{0}^{1}\left[\kappa\left(\frac{T_{x}}{T}\right)^{2}+\frac{m \rho v^{2}}{T \tau_{p}}-\frac{e \rho v}{T} \varphi_{x}\right] d x \quad(\text { by }(2))=
\end{aligned}
$$




$$
\begin{aligned}
=\frac{3}{2} & \frac{d}{d t} \int_{0}^{1} \rho(\ln \rho+\ln T) d x-\int_{0}^{1}\left[\kappa\left(\frac{T_{x}}{T}\right)^{2}+\frac{m \rho v^{2}}{T \tau_{p}}-\frac{e \rho v}{T} \varphi_{x}\right] d x \\
& +\frac{5}{2} \int_{0}^{1} \rho v_{x} d x \quad(\text { by }(1)) .
\end{aligned}
$$

Thus,

$$
\begin{aligned}
& \frac{2}{5} \int_{0}^{1} \rho(\ln \rho+\ln T) d x-\frac{4}{15} \int_{0}^{t} \int_{0}^{1}\left[\kappa\left(\frac{T_{x}}{T}\right)^{2}+\frac{m \rho v^{2}}{T \tau_{p}}\right] d x d t \\
= & \frac{2}{5} \int_{0}^{1} \rho_{0}\left(\ln \rho_{0}+\ln T_{0}\right) d x-\frac{2}{15} \int_{0}^{t} \int_{0}^{1} \frac{3 n\left(T-T^{(0)}\right)+m \rho v^{2}}{T \tau_{w}} d x d t \\
& -\frac{2}{3} \int_{0}^{t} \int_{0}^{1} \rho v_{x} d x d t .
\end{aligned}
$$

Subtracting (96) from (95), we obtain

$$
\begin{aligned}
& \int_{0}^{1} \frac{1}{m}\left(3 \rho \ln T+2 \rho \ln \frac{1}{\rho}\right) d x \\
& \quad-\frac{1}{m} \int_{0}^{t} \int_{0}^{1}\left[2 \kappa\left(\frac{T_{x}}{T}\right)^{2}+\frac{m \rho v^{2}\left(2 \tau_{w}-\tau_{p}\right)}{T \tau_{p} \tau_{w}}-\frac{3 \rho\left(T-T^{(0)}\right)}{T \tau_{w}}\right] d x d t \\
& =\quad \int_{0}^{1} \frac{1}{m}\left(3 \rho_{0} \ln T_{0}+2 \rho_{0} \ln \frac{1}{\rho_{0}}\right) d x .
\end{aligned}
$$

Subtracting (97) from (93), we obtain the following inequality:

$$
\begin{aligned}
\frac{3}{m} \int_{0}^{1} \rho(T- & -\ln T) d x+\frac{2}{m} \int_{0}^{1} \rho\left(\frac{1}{\rho}-1-\ln \frac{1}{\rho}\right) d x \\
& +2 \int_{0}^{t} \int^{1}\left(\frac{\kappa}{m}\left(\frac{T_{x}}{T}\right)^{2}+\varepsilon v_{x}^{2}\right) d x d t+\frac{e}{m} f(t)+\int_{0}^{1} \rho v^{2} d x \\
& +\int_{0}^{t} \int_{0}^{1}\left[\frac{3 \rho\left(T-T^{(0)}\right)}{m \tau_{w}}+\frac{\rho v^{2}}{\tau_{w}}\left(1+\frac{2 \tau_{w}-\tau_{p}}{T \tau_{p}}\right)\right] d x d t \\
=\int_{0}^{1} \frac{\rho_{0}}{m}\left[3 \left(T_{0}-\right.\right. & \left.\left.\ln T_{0}\right)+2 \ln \rho_{0}+m v_{0}^{2}-5\right] d x+\int_{0}^{t} \int_{0}^{1} \frac{3 \rho\left(T-T^{(0)}\right)}{m T \tau_{w}} d x d t
\end{aligned}
$$




$$
\begin{aligned}
\leq \frac{1}{m} \int_{0}^{1} \rho_{0}\left[3 \left(T_{0}\right.\right. & \left.\left.+\left|\ln T_{0}\right|\right)+2\left|\ln \rho_{0}\right|+m v_{0}^{2}+5\right] d x \\
& +\frac{1}{T^{(0)}} \int_{0}^{1} \rho_{0}\left(\frac{3}{m} T_{0}+v_{0}^{2}\right) d x \quad(\text { by }(93)) .
\end{aligned}
$$

It follows that

$$
\int_{0}^{1}\left(\frac{T_{x}}{T}\right)^{2} d x \in L^{1}(0, \infty)
$$

From boundary value conditions, we have

$$
\begin{aligned}
\frac{d}{d t} \int_{0}^{1} v_{x}^{2} d x & =-2 \int_{0}^{1} v_{x x} v_{t} d x \\
\frac{d}{d t} \int_{0}^{1}(\ln T)_{x}^{2} & =-2 \int_{0}^{1}\left(\frac{T_{x x}}{T^{2}}-\frac{T_{x}^{2}}{T^{3}}\right) T_{t} d x .
\end{aligned}
$$

Then, it follows from Lemma 6 that

$$
\int_{0}^{\infty}\left(\left|\frac{d}{d t}\left\|v_{x}\right\|_{L^{2}(0,1)}^{2}(t)\right|+\left|\frac{d}{d t}\left\|(\ln T)_{x}\right\|_{L^{2}(0,1)}^{2}(t)\right|\right) d t \leq C .
$$

Thus, $\left\|v_{x}\right\|_{L^{2}}^{2}(t)$ and $\left\|(\ln T)_{x}\right\|_{L^{2}}^{2}(t)$ are uniformly continuous on $[0, \infty)$. (94) and (98) imply that

$$
\begin{aligned}
\left\|v_{x}\right\|_{L^{2}(0,1)}^{2}(t) & \rightarrow 0, \quad t \rightarrow \infty \\
\left\|(\ln T)_{x}\right\|_{L^{2}(0,1)}^{2}(t) & \rightarrow 0, \quad t \rightarrow \infty
\end{aligned}
$$

Therefore,

$$
\begin{aligned}
v^{2}(x, t) & =\left(\int_{0}^{x} v_{x} d x\right)^{2} \leq\left\|v_{x}\right\|_{L^{2}(0,1)}^{2}(t) \rightarrow 0, \quad t \rightarrow \infty, \\
\left(\ln \frac{T}{T^{(0)}}\right)^{2}(x, t) & =\left(\int_{0}^{x}(\ln T)_{x} d x\right)^{2} \leq\left\|(\ln T)_{x}\right\|_{L^{2}(0,1)}^{2}(t) \rightarrow 0, \quad t \rightarrow \infty .
\end{aligned}
$$

Finally, (8) and (11) follow easily from the integrations of the equations (1) and (91), respectively.

This completes the proof of Theorem 2 . 


\section{REFERENCES}

[1] G. Baccarani And M. R. Wordeman, An investigation of steady-state velocity overshoot effects in $S i$ and GaAs devices. Solid State Electronics 28 (1985), 404-416.

[2] K. BLØTEKJÆR, Transport equations for electrons in two-valley semiconductors. IEEE Trans. Electron Devices, ED-17 (1970), 38-47.

[3] G.-Q. Chen, Global solutions to the compressible Navier-Stokes equations for a reacting mixture. SIAM J. Math. Anal., 23 (1992), 609-634.

[4] C. M. DAFermos, Global smooth solutions to the initial-boundary value problem for the equation of the one-dimensional nonlinear thermoviscoelasticity. SIAM J. Math. Anal., 13 (1982), 397-408.

[5] C. M. Dafermos, Estimates for conservation laws with little viscosity. SIAM J. Math. Anal., 18 (1987), 409-421.

[6] E. FAtemi, J. Jerome, And S. Osher, Solution of the hydrodynamic device model using high-order non-oscillatory shock capturing algorithms. IEEE Transactions on Computer-Aided Design of Integrated Circuits and Systems, 10 (1991), 232244.

[7] A. Friedman, Partial Differential Equations of Parabolic Type. Prentice Hall, 1964.

[8] I. M. GAmBA, Stationary transonic solutions of a one-dimensional hydrodynamic model for semiconductors. Commun. in P.D.E., 17 (1992), 553-577.

[9] C. L. GARDNER, Numerical simulation of a steady-state electron shock wave in a submicron semiconductor device. IEEE Transactions on Electron Devices, 38 (1991), 392-398.

[10] C. L. GARDNER, Hydrodynamic and Monte Carlo simulation of an electron shock wave in a one micron semiconductor device. In Proceedings of the International Workshop on Computational Electrons, University of Illinois at UrbanaChampaign, (1992). Beckman Institute.

[11] C. L. Gardner, J. Jerome, and D. J. Rose, Numerical methods for the hydrodynamic model: subsonic flow. IEEE Trans. Computer-Aided Design, 8 (1989), 501-507.

[12] D. Hoff, Global well-posedness of the Cauchy problem for the Navier-Stokes equations of nonisentropic flow with dicontinuous initial data. J. Diff. Eqs., 95 (1992), 33-74.

[13] N. ITAYA, A survey on generalized Buger's equation with a pressure model term. J. Math. Kyoto Univ., 16 (1976), 223-240.

[14] Y. I. KANEL, On a model system of equations of one-dimensional gas motion. Differential Equations, 4 (1968), 374-380.

[15] A. V. KAZHIKHov, Stabilization of solutions of an initial-boundary-value problem for the equations of motion of a barotropic viscous fluid. Differential Equations, 15 (1979), 463-467.

[16] S. N. KRUŽKov, A priori estimates for the derivative of a solution of a parabolic equation and some of its applications. Soviet Math. Dokl., 7 (1966), 1215-1218.

[17] O. A. Ladyženskaya, V. A. Solonnikov, and N. N. Ural'Ceva, Linear and Quasilinear Equations of Parabolic Type, volume 23 of Translations of Mathematical Monographs. American Mathematical Society, Providence, 1968.

[18] T. NishidA, Equations of motion of compressible viscous fluid. In Pattens and Waves-Qualitative Analysis of Nonlinear Differential Equations, Nishida T., Mimura M., and Fujii H. (eds.), . North-Holland, 1986. 
[19] M. Rudan, F. Odeh, AND J. White, Numerical solution of the hydrodynamic model for a one-dimensional semiconductor. COMPEL (The International Journal for Computation and Mathematics in Electronic Engineering), 6 (1987), 151-170.

[20] A. TANI, On the first initial-boundary value problem of the generalized Burger's equation. Publ. RIMS Kyoto Univ., 10 (1974), 209-233.

[21] B. Zhang, Convergence of the Godunov scheme for a simplified one-dimensional hydrodynamic model for semiconductor devices. Commun. Math. Phys., 157 (1993), 1-22.

Department of Mathematics

Stanford University

Stanford, California 94305

Received: April 10th, 1995. 